\title{
AN INTERCOMPARISON \\ OF MEDIUM ENERGY CROSS-SECTION CODES
}

\author{
Sol Pearlstein
}

May 1988

IMFORMATION AMALYSIS CENTER REPORT

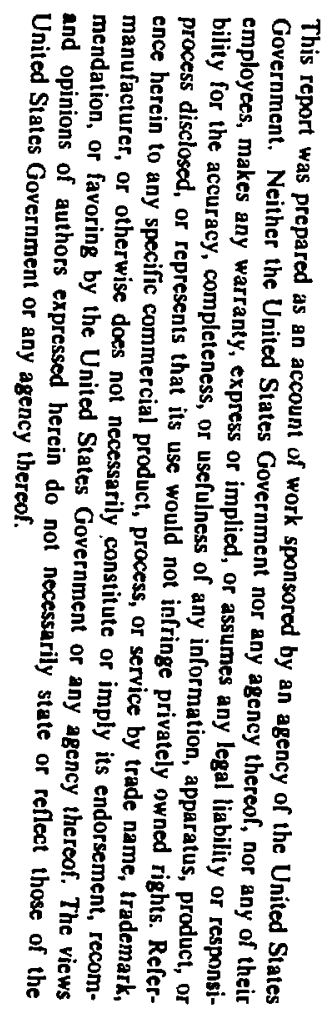

MATIONAL MULLEAR DATA CENTEB BROOKHAVEN MATIONAL LABORATORY

ASSOCIATED UNIVERSITIES, INC. UNOER CONTRACT NO. DE-AGO2-76CHOOO16 WITH THE UNITED STATES DEPARTMENT OF ENERGY

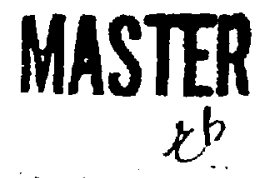




\section{DISCLAIMER}

This report was prepared as an account of work sponsored by an agency of the United States Government. Neither the United States Government nor any agency thereof, nor any of their employees, nor any of their contractors, subcontractors, or their employees, makes any warranty, express or implied, or assumes any legal liability or responsibility for the accuracy, completeness, or usefulness of any information, apparatus, product, or process disclosed, or represents that its use would not infringe privately owned rights. Reference herein to any specific commercial product. process. or service by trade name, trademark, manufacturer, or otherwise, does not necessarily constitute or imply its endorsement, recommendation, or favoring by the United States Government or any agency. contractor or subcontractor thereof. The views and opinions of suthors expressed herein do not necessarily state or reflect those of the United States Government or any agency, contractor or aubcontractor thereof.

Printed in the United States of America

Available from

National Technical Information Service

U.S. Department of Commerce

Spring field, VA 22161

NTIS price codes:

Printeci Copy: A04; Microfiche Copy: A01 


\section{TABLE OF CONTENTS}

Page No.

I. Benchmark Calculations...............................

II $\quad$ Benchmarks $\ldots \ldots \ldots \ldots \ldots \ldots \ldots \ldots \ldots \ldots \ldots \ldots \ldots \ldots \ldots \ldots \ldots \ldots$

III. Code Results Submitted.............................

III.1 Activation Yields...........................

III.2 Neutron Emission Double Differential Cross-Sections......11

III.3 Calculation Times.............................

IV. $\quad$ Discussion..................................... 14

IV.1 Activation Yields...........................

IV.2 Neutron Emission Double Differential Cross-Sections......15

IV.3 Calculation Times..........................

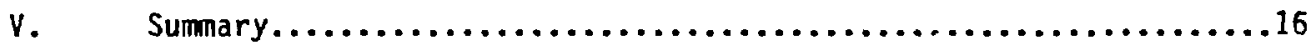

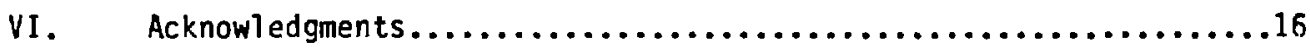

References $\ldots \ldots \ldots \ldots \ldots \ldots \ldots \ldots \ldots \ldots \ldots \ldots \ldots \ldots \ldots \ldots \ldots \ldots \ldots \ldots \ldots$

Appendix I - Proposed MENDWG Benchmark Problem....................18

Appendix II - Code Abstracts................................19

II.1 PNEM................................... 19

II $.2 \quad$ ALICE....................................

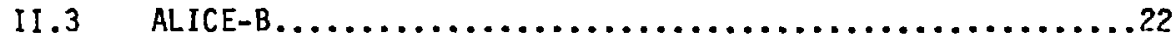

II.4 HETC-CCC-178............................23

II.5 HETC-H Description and Calculational Details..........26

II.6 HETC $-F($ TIERCE) $\ldots \ldots \ldots \ldots \ldots \ldots \ldots \ldots \ldots \ldots \ldots \ldots \ldots \ldots \ldots$

Figures.................................................. 


\section{Abstract}

Five medium energy proton reaction cases are selected for benchmarking nuclear model codes. The quantities calculated are isotopic activation yields for $180 \mathrm{MeV}$ protons on Ai and 40-200 $\mathrm{MeV}$ protons on $\mathrm{Co}$, and double differential neutron emission spectra from Al, $\mathrm{Zr}-90$ and $\mathrm{Pb}-208$ for $35,80,160,318$, and 800 MeV proton bombardment. The five codes for which results are presented consist of three types: (1) a closed form preequilibrium plus evaporation model, (2) an intranuclear-cascade and evaporation model, and (3) a model relying on nuclear systematics. The characteristics of each code are described. There are orders of magnitude differences in the time for each type of code to calculate neutron emission spectra, with codes using systematics, preequilibrium and intranuclear-cascade models requiring seconds, minutes and hours, respectively. Calculations are not compared with experiment in this initial study.

For double differential neutron emission spectra, there is good overall agreement in magnitude among the different types of codes at forward ang?es. Differences where they occur at forward angles are greatest for the mid-energy neutrons emitted. At back angles the incident energy at which the best overall agreement is obtained is $160 \mathrm{MeV}$ and the material for which the hest overall agreement is obtained is Al.

I.

Benchmark Calculations

If ultimately the results of benchmark experiments of medium energy applications can be simulated using nuclear cross-section codes and radiation transport methods, engineering designs can be achieved relatively quickly and inexpensively with fewer experiments needed. Nuclear model code calculations can be compared to some nuclear cross-section measurements that are available but measurenients of the same cross-section by different authors do not always converge to the same values. Similarly, different nuclear model codes do not always converge to the same value. It is helpful to understand what the cross-section codes themselves are doing. This report does not compare calculation with experiment. Instead, it compares the results from different codes calculating the same problem description. The calculational benchmarks chosen were of the type to anticipate experimental results that may be forthcoming.

It is hoped that this report will be part of a long range study to validate codes for medium energy applications. Extensions of this work will include comparisons with experimental data. Only partial results have been received to date but are considered sufficient to (1) provide information about the capability of codes, (2) compare the results abtained from different codes using similar input but different algorithms (or even ostensibly the same algorithms) for solution and (3) stimulate more contributions and additional benchmarks. 
I I.

Benchmarks

The benchmark specifications for an intercomparison of cross-section codes were formulated at a May 1987 meeting of the Medium Energy Nuclear Data Working Group. At that meeting a Codes and Models Subgroup chaired by M. Blann proposed the following five benchmarks:

Activation Yields

1. $180 \mathrm{MeV}$ protons on AT-27

2. 40-200 MeV protons on Co-59

Neutron Emission Spectra

$35,80,160,318$ and $800 \mathrm{MeV}$ protons on

3. $\quad A 1-27$

4. $2 r-90$

5. $\mathrm{Pb}-208$

Appendix I.

The specifications for the 5 benchmark problems are given in

The benchmark problems were limited in scope so that the comparison of the results would not be too cumbersome, time consuming or error prone. Fortunately, most results were received in computerized form greatly reducing the labor involved and the chances for errors.

III. Code Results Submitted

The codes for which results were received consisted of two types of algorithm solutions, (1) deterministic, for which a given set of input produces a unique answer, and (2) Monte Carlo, for which a given set of input produces a statistically determined answer whose convergence improves as the number of trials is increased.

The cross section model codes for which results were received consisted of three types, (1) a closed form preequilibrium plus evaporation model, (2) an intranuclear-cascade and evaporation model, and (3) a model relyigg on nuclear systematics. An example of the first type of code is ALICE ${ }^{2}$ wich uses Weisskopf-Ewing evaporation, Bohr-Wheeler rotating drop fission competition and the exciton preequilibrium models to describe nuclear reactions. Examples of the second type of code are various versions of the high energy transport code HETC which are extensions of the Bertini model. Both ALICE and HETC attempt to physically model nuclear reactions and rely on very few empirically determined parameters. An example of the third type of code is PNEM which uses semiphysical evaporation shapes to fit experimental neutron emission spectra from proton induced reactions. ${ }^{4}$ These data are observed to vary nearly monotonically with increasing energy, mass, and angle. The PNEM parameters were empirically determined for the energy range 
about $590 \mathrm{MeV}$, so that the agreement between calculation and experiment should be quite good, but that is not the subject of this first stage investigation. Unlike the other codes discussed here which calculate a wide variety of nuclear cross-sections, PNEM mainly calculates the double differential neutron emission cross-section.

The results from five codes are discussed in this report.

1. ALICE, submi.tted by Marshall Blann, Lawrence Livermore National Laboratory

2. ALICE-B, submitted by Sol Pearlstein, Brookhaven National Laboratory

3. HETC-F (TIERCE), submitted by Claude Philis, CEA, Bruyeres-leChatel

4. HETC-H (HETC-KFA-2), submitted by Detlef Filges, KFA, Jeulich

5. PNEM, submitted by Sol Pearlstein, Brookhaven National Laboratory

Append ix II.

Brief descriptions of each code were requested and are contained in

In a "blind" benchmark comparison the resuits would be published as received. In this initial study, a draft report was circulated to the submitters so that they could confirm that their data had been used correctly. An error was found in the HETC-H calculations and renormalized results were subsequently received and used for the comparison. An example of these changes is given in Section III.2.

\section{III.1 Activation Yields}

Tabulated results were received from $M$. Blann using ALICE and computation formatted results from $S$. Pearlstein using ALICE-B. Although results in computerized form had not been required it was soon evident that computerized results greatly facilitated the comparison. It was noted that the Blann and Pearlstein results differed hecause Blann had chosen the normal pairing shift $(M P=3)$ and 3 output particles $(M 3=3)$ and Pearlstein had chosen the level density ground state shift with shell corrections included (MP=2) and 4 output particles $(M 3=4)$. For identical input the two codes produced identical results to 5 significant figures except for small differences where partial waves for impact angular momenta greater than 21 (a somewhat arbitrary present limit in ALICE but not in ALICE-B) were involved. On the one hand the exact agreenent is not surprising because $A L I C E \cdots B$ was derived from ALICE but on the other hand ALICE-B has several enhancements and has been adapted to run on a DEC-10 and VAX-780, with 36 and 32 bit words, respectively, whereas ALICE results are obtained on a $C D C-7600$, with $60 . b i t$ words. 
Partial wave requirements for angular momenta greater than 21 are negligible for $180 \mathrm{MeV}$ protons on $\mathrm{Al}-2 \%$ and protons below $140 \mathrm{MeV}$ on Co-59. Therefore, the computation format output of ALICE-B which is essentially equivalent to those of ALICE, was used to facilitate the presentation of results. Also, since the differences between ALICE and ALICE-B Calculations resulted from choices in input parameters rather than differences in the codes, it is useful to compare the effect of these input parameter choices, and others, on the results to illustrate their effect to present and potential users of the ALICE code.

Table I.

For Benchmarks 1 and 2 the parameters used by Blann are shown in

\section{Table I. ALICE Input Parameters for Benchmarks 1 and 2}

$N A=11$; The number of isotopes included for each residual element.

$N Z=9$; The number of elements included in the cascade calculations.

$M C=10 ;$ Atomic masses option.

$M P=3$; Normal pairing shift option, 0 for odd-even nuclei, delta added to excitation for odd-odd nuclei.

INVER $=2$; Inverse cross-sections calculated by sharp cutoff method.

$M 3=3$; Neutrons, protons, and alphas emitted.

For Benchmark 1, $180 \mathrm{MeV}$ protons on A1-27, the cross-section yields of activation products are shown in Figure 1 and 1 isted in Table II.

For Benchmark 2, 40 to $200 \mathrm{MeV}$ protons on Co-59, the cross-section yields of activation products at $20 \mathrm{MeV}$ steps (received in $10 \mathrm{MeV}$ steps) are listed in Table III. Graphical displays at 120, 160, and $200 \mathrm{MeV}$ are shown in Figure 2 .

For Benchmark 2, the ALICE options listed in Table IV were used for calculations. The activation cross-sections for Co-56, Mn-54, and V-50 using Table IV options are shown in Figure 3. 
Table II. Yield Cross-Section of Activation Products from $180 \mathrm{MeV}$ Protons on A1-27

YIELD CROSS-SECTION (MB)

\begin{tabular}{|c|c|c|c|c|c|c|c|c|c|c|c|}
\hline$Z \backslash A$ & $\stackrel{28}{000 E+00}$ & $\begin{array}{c}27 \\
0.4 D 4 E+01\end{array}$ & $\begin{array}{c}26 \\
.182 E+01\end{array}$ & $\begin{array}{c}25 \\
0.201 E-01\end{array}$ & $0.3^{24}$ & $\begin{array}{c}23 \\
0.552 E-04\end{array}$ & $\frac{22}{0.297 \mathrm{E}-06}$ & $\frac{21}{0.455 E-08}$ & $\frac{20}{0.000 E+\infty 0}$ & $\stackrel{19}{0.000 f+00}$ & $\begin{array}{l}18 \\
.000 E+0\end{array}$ \\
\hline 13 & $0.7^{27}$ & $\begin{array}{r}26 \\
0.1856\end{array}$ & $\begin{array}{r}25 \\
.533\end{array}$ & $\begin{array}{r}24 \\
.312 \mathrm{E}\end{array}$ & 0.11 & 03 & 0.64 & -07 & & & Doul \\
\hline 12 & 8) & .2616 & $\begin{array}{r}24 \\
379\end{array}$ & .23 & $\begin{array}{r}22 \\
0.469\end{array}$ & .113 & .33 & 55 & .42 & 11 & .00 \\
\hline 11 & $\begin{array}{c}25 \\
398 E+00\end{array}$ & $.243 E+01$ & 238 & $\stackrel{22}{22} .321 E+02$ & $0.355 E$ & +00 & .56 & $\begin{array}{r}18 \\
409\end{array}$ & & & .56 \\
\hline 10 & $\stackrel{24}{135 E-01}$ & $\stackrel{23}{198 E+00}$ & $\begin{array}{c}22 \\
245 E\end{array}$ & $\stackrel{21}{.150 E+02}$ & $\begin{array}{r}20 \\
.134 \mathrm{E}\end{array}$ & $\begin{array}{r}19 \\
.199\end{array}$ & $\begin{array}{r}18 \\
.595\end{array}$ & $\begin{array}{r}17 \\
0.212\end{array}$ & 0.000 & 27 & $0.000 E$ \\
\hline 9 & $\begin{array}{l}23 \\
100 E-02\end{array}$ & $\begin{array}{c}22 \\
773 E\end{array}$ & 0.921 & $\begin{array}{r}20 \\
867\end{array}$ & $\begin{array}{r}19 \\
.357\end{array}$ & $\begin{array}{r}18 \\
.592\end{array}$ & 0 & 11 & 03 & 15 & $306 \mathrm{E}-$ \\
\hline 8 & ${ }_{798 E-O S}^{22}$ & $\begin{array}{c}21 \\
125 \mathrm{E}\end{array}$ & $\begin{array}{c}20 \\
0.260 E-02\end{array}$ & $\begin{array}{l}19 \\
311 E-0\end{array}$ & $\begin{array}{r}18 \\
.197\end{array}$ & $\begin{array}{c}17 \\
0.202 E+c\end{array}$ & $\begin{array}{c}16 \\
182 E+C\end{array}$ & $\begin{array}{r}15 \\
.257\end{array}$ & .13 & 4 & 12 \\
\hline 7 & ${ }_{552 E-07}^{21}$ & $\stackrel{20}{353 E-06}$ & $\begin{array}{r}19 \\
.2696\end{array}$ & $\begin{array}{r}18 \\
283 E\end{array}$ & $\begin{array}{r}17 \\
.652 E\end{array}$ & $\frac{16}{0.143 E+\infty 0}$ & $\begin{array}{r}15 \\
0.483\end{array}$ & $\begin{array}{r}14 \\
.655\end{array}$ & $\begin{array}{c}13 \\
0.704 E\end{array}$ & 0.22 & $\frac{11}{0.127 \mathrm{E}-\mathrm{C}}$ \\
\hline 6 & $120 E-10$ & $\frac{19}{424 E-10}$ & $\begin{array}{l}18 \\
.718 E-07\end{array}$ & 05 & 34 & 02 & $E-01$ & 11 & $E+01$ & $3 E+00$ & 30 \\
\hline
\end{tabular}


Table III. Yield Cross-Sections of Activation Products from Protons on Ca-59

IIIa. $40 \mathrm{MeV}$ Yield Cross-Section (MB)

YIELD CROSS-SECTION (MB)

\begin{tabular}{|c|c|c|c|c|c|c|c|c|c|c|c|}
\hline $\begin{array}{l}219 \\
28\end{array}$ & $\begin{array}{c}60 \\
0.000 E+00\end{array}$ & $\begin{array}{c}59 \\
0.220 E+02\end{array}$ & $\begin{array}{c}58 \\
0.442 E+02\end{array}$ & $\begin{array}{c}57 \\
0.418 E+02\end{array}$ & $\begin{array}{c}56 \\
0.220 E+02\end{array}$ & $\begin{array}{c}55 \\
0.201 E-01\end{array}$ & $\frac{54}{0.000 E+\infty 0}$ & $\begin{array}{c}53 \\
0.000 £+00\end{array}$ & $0.000 E+\infty 0$ & $\stackrel{51}{0.000 E+00}$ & $\stackrel{50}{0.000 E+\infty}$ \\
\hline 27 & $\frac{59}{0.421 E+02}$ & $\begin{array}{c}58 \\
0.809 E+02\end{array}$ & $\begin{array}{c}57 \\
0.132 E+03\end{array}$ & $\begin{array}{c}56 \\
0.211 E+03\end{array}$ & $\begin{array}{c}55 \\
0.352 E+02\end{array}$ & $\begin{array}{c}54 \\
0.000 E+00\end{array}$ & $\begin{array}{c}53 \\
0.000 E+\infty\end{array}$ & $0.000 E+00$ & $\stackrel{51}{0.000 E+00}$ & 100 & $\begin{array}{c}49 \\
0.000 E+\infty 0\end{array}$ \\
\hline 26 & $\begin{array}{c}58 \\
0.134 E+02\end{array}$ & $\begin{array}{c}57 \\
0.202 E+02\end{array}$ & $\begin{array}{c}56 \\
0.595 E+02\end{array}$ & $\begin{array}{c}55 \\
0.547 E+02\end{array}$ & $\stackrel{54}{0.109 \mathrm{E}+02}$ & $\begin{array}{c}53 \\
0.139 E+02\end{array}$ & $\begin{array}{c}52 \\
0.25 \mathrm{BE}-01\end{array}$ & $\frac{51}{0.000 E+\infty 0}$ & $\stackrel{50}{0.000 E+\infty}$ & $\begin{array}{c}49 \\
0.000 E+00\end{array}$ & $\begin{array}{c}48 \\
0.000 E+\infty\end{array}$ \\
\hline 25 & $\begin{array}{c}57 \\
0.138 E+00\end{array}$ & $\begin{array}{c}56 \\
0.900 E+00\end{array}$ & $\begin{array}{c}55 \\
0.145 E+01\end{array}$ & $\begin{array}{c}54 \\
0.920 E+01\end{array}$ & $\begin{array}{c}53 \\
0.3 B 6 E+02\end{array}$ & $\begin{array}{c}52 \\
0.176 E+01\end{array}$ & $\begin{array}{c}51 \\
0.000 E+00\end{array}$ & $\begin{array}{c}50 \\
0.000 E+\infty 0\end{array}$ & $0.000 t+00$ & $\frac{48}{0.000 E+\infty 0}$ & $0.000 E+\infty 0$ \\
\hline 24 & $0.213 E-03$ & $\begin{array}{c}55 \\
0.269 E-04\end{array}$ & $143 E+\infty$ & $\begin{array}{c}53 \\
0.168 E+01\end{array}$ & $.367 E+\infty 0$ & $\begin{array}{c}51 \\
0.405 E+\infty\end{array}$ & $\stackrel{50}{0.994 E+\infty}$ & $\frac{49}{0.234 E-04}$ & $0.000 E+00$ & $\stackrel{47}{0.000 E+\infty}$ & $\begin{array}{c}46 \\
0.000 E+\infty 0\end{array}$ \\
\hline 23 & $\begin{array}{c}55 \\
0.000 E+\infty 0\end{array}$ & $\begin{array}{c}54 \\
0.000 E+00\end{array}$ & $\begin{array}{c}53 \\
0.566 E-03\end{array}$ & $\begin{array}{c}52 \\
0.500 \varepsilon-05\end{array}$ & $\frac{51}{0.108 \mathrm{E}+00}$ & $\begin{array}{c}50 \\
0.277 E+\infty 0\end{array}$ & $\stackrel{49}{0.123 E-03}$ & $\frac{48}{0.000 E+00}$ & $0.000 E+\infty$ & $\stackrel{46}{0.000 E+00}$ & $0.000 E+\infty 0$ \\
\hline 22 & $\frac{54}{0.000 E+\infty 0}$ & $\stackrel{53}{0.000 E+00}$ & .52 & $0.000 \varepsilon+00$ & $\stackrel{50}{0.125 E-02}$ & $\stackrel{49}{.177 \mathrm{E}-07}$ & $\stackrel{48}{0.566 E-02}$ & $\stackrel{47}{0.490 \varepsilon-02}$ & $0.000 E+00$ & $0.000 E+\infty 0$ & $0.000 E+\infty 0$ \\
\hline 21 & $0.000 E+\infty 0$ & $\begin{array}{c}52 \\
0.000 E+\infty 0\end{array}$ & $0.000 \varepsilon+\infty$ & $\frac{50}{0.000 \varepsilon+00}$ & $\stackrel{49}{0.000 E+00}$ & $0.000 E+\infty$ & $\stackrel{47}{148 E-04}$ & $0.000 E+\infty 0$ & $0.000 E+00$ & $0.000 E+\infty$ & $0.000 E+\infty$ \\
\hline 20 & $0.000 \varepsilon+\infty$ & $\frac{51}{0.000 E+00}$ & $\begin{array}{l}50 \\
000 E+00\end{array}$ & $0.000 E+\infty 0$ & $0.000 E+00$ & $.000 E+\infty 0$ & $\begin{array}{r}46 \\
0.000\end{array}$ & $0.000 \epsilon+\infty$ & 7 & $.000 E+\infty 0$ & $0.000 E+\infty 0$ \\
\hline
\end{tabular}

IIIb. $\quad 60 \mathrm{MeV}$ Yield Cross-Section (MB)

YIELD CROSS-SECTION (MB)

\begin{tabular}{|c|c|c|c|c|c|c|c|c|c|c|c|}
\hline 28 & $\frac{60}{0.000 \varepsilon+\infty}$ & $\stackrel{59}{0.292 \mathrm{E}+02}$ & $\begin{array}{c}58 \\
0.851 E+02\end{array}$ & $\begin{array}{l}57 \\
0.190 E+03\end{array}$ & $\stackrel{56}{0.693 E+\infty}$ & $\stackrel{55}{0.000 E+\infty 0}$ & $0.000 E+\infty$ & $0.000 E+\infty 0$ & $0.000 \kappa+\infty$ & $0.000 E+\infty$ & $\stackrel{50}{0.000 E+\infty 0}$ \\
\hline 27 & $\begin{array}{c}59 \\
0.530 E+C 2\end{array}$ & $\begin{array}{c}58 \\
0.118 E+03\end{array}$ & $\begin{array}{c}57 \\
0.348 E+03\end{array}$ & $\begin{array}{c}56 \\
0.489 E+01\end{array}$ & $\frac{55}{0.000 €+\infty 0}$ & $\frac{54}{0.000 \mathrm{E}+\infty 00}$ & $\frac{53}{0.000 E+\infty 0}$ & $\frac{52}{0.000 E+\infty 0}$ & $\frac{51}{0.000 E+\infty 0}$ & $\begin{array}{c}50 \\
0.000 E+0 . ?\end{array}$ & $\frac{49}{0.000 E+\infty}$ \\
\hline 26 & $\begin{array}{c}56 \\
0.130 E+02\end{array}$ & $\begin{array}{c}57 \\
0.253 \mathrm{E}+02\end{array}$ & $\begin{array}{c}56 \\
0.226 E+01\end{array}$ & $\begin{array}{c}55 \\
.896 E+01\end{array}$ & $\frac{54}{0.472 E+02}$ & $\frac{53}{1.000 E+\infty 00}$ & $0.000 E+\infty 0$ & $\frac{51}{0.000 E+\infty}$ & $0.000 E+\infty 0$ & $0.000 E+00$ & $0.000 E+\infty$ \\
\hline 25 & $\stackrel{57}{0.159 E-01}$ & $\begin{array}{c}56 \\
0.000 E+\infty\end{array}$ & $\frac{55}{0.325 E+01}$ & $0.122 E+02$ & $\frac{53}{0.000 E+\infty 0}$ & $0.000 E+\infty$ & $\begin{array}{c}51 \\
0.000 E+00\end{array}$ & $\begin{array}{c}50 \\
0.000 \varepsilon+00\end{array}$ & $0.000 E+\infty 0$ & $0.000 E+00$ & $\frac{47}{0.000 E+\infty 0}$ \\
\hline 24 & $0.000 E+\infty 0$ & $\frac{55}{0.000 \varepsilon+00}$ & $\begin{array}{c}\mathbf{5 4} \\
0.225 \mathrm{E}-01\end{array}$ & $\frac{53}{0.000 E+\infty 0}$ & $\begin{array}{c}52 \\
0.506 E+\infty 0\end{array}$ & $\begin{array}{c}51 \\
0.460 E+\infty\end{array}$ & $\begin{array}{c}50 \\
0.000 E+\infty\end{array}$ & $0.000 E+\infty$ & $0.000 E+\infty$ & $0.000 E+\infty 0$ & $0.000 E+\infty 0$ \\
\hline 23 & $\frac{55}{0.000 E+\infty 0}$ & $\frac{54}{0.000 \varepsilon+00}$ & $0.000 E+\infty$ & $.000 E+\infty$ & $\frac{51}{0.713 E-02}$ & $\frac{50}{0.000 E+\infty}$ & $0.000 E+\infty$ & $0.000 E+\infty 0$ & $0.000 E+\infty 0$ & $0.000 \varepsilon+\infty 0$ & $\stackrel{45}{0.000 E+\infty 0}$ \\
\hline 22 & $0.000 E+\infty 0$ & $\begin{array}{c}53 \\
0.000 E+\infty 0\end{array}$ & $0.000 E+\infty 0$ & $\frac{51}{0.000 E+\infty 0}$ & $0.000 \mathrm{50}+\infty$ & $\stackrel{49}{0.000 E+\infty 0}$ & $0.889 E-06$ & $0.000 E+00$ & $0.000 E+\infty 0$ & $0.000 E+\infty 0^{45}$ & $0 .{ }^{44}$ \\
\hline 21 & $\frac{53}{0.000 E+\infty}$ & $\frac{52}{0.000 \varepsilon+\infty 0}$ & $0.000 \varepsilon+\infty$ & $\begin{array}{c}50 \\
0.000 E+\infty\end{array}$ & $0.000 E+\infty 0$ & $\stackrel{48}{48}_{0.000+\infty}$ & $0.000 E+\infty$ & $0.000 E+00$ & $0.000 E+\infty 0$ & $0.000 E+\infty 0$ & $\stackrel{43}{0.000 E+\infty}$ \\
\hline 20 & $\frac{52}{0.000 E+\infty}$ & $\frac{51}{0.000 E+\infty}$ & $\begin{array}{c}50 \\
0.000 E+\infty\end{array}$ & $\stackrel{49}{49}^{400 E+00}$ & $0.000<+\infty 0$ & 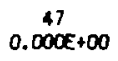 & $0.000 E+\infty 0$ & $0.000 \varepsilon+00$ & $0.000 E+\infty$ & $0.000 E+\infty 0$ & $0.000 E+\infty 0$ \\
\hline
\end{tabular}


YIELO CROSS-SECTION (ME)

\begin{tabular}{|c|c|c|c|c|c|c|c|c|c|c|c|}
\hline $\begin{array}{r}21 A \\
28\end{array}$ & $\begin{array}{c}60 \\
0.000 E+00\end{array}$ & $\begin{array}{c}59 \\
0.165 E+02\end{array}$ & $\begin{array}{c}58 \\
0.264 E+02\end{array}$ & $\begin{array}{c}57 \\
0.224 E+02\end{array}$ & $\begin{array}{c}56 \\
0.840 E+01\end{array}$ & $\begin{array}{c}55 \\
0.376 E * 00\end{array}$ & $\begin{array}{c}54 \\
0.112 \mathrm{E}-02\end{array}$ & $\begin{array}{c}53 \\
0.000 E+00\end{array}$ & $\frac{52}{0.000 E+\infty 00}$ & $\frac{51}{0.000 E+00}$ & $\begin{array}{c}50 \\
0.000 E+00\end{array}$ \\
\hline 27 & $\begin{array}{l}59 \\
0.320 E+02\end{array}$ & $\begin{array}{c}58 \\
.659 \mathrm{gE}+02\end{array}$ & $\begin{array}{l}57 \\
0.910 E+02\end{array}$ & $\begin{array}{c}56 \\
.112 \mathrm{E}+03\end{array}$ & $\begin{array}{c}55 \\
0.630 E+02\end{array}$ & $\begin{array}{c}54 \\
0.313 \mathrm{E}+01\end{array}$ & $\begin{array}{c}53 \\
0.126 E-03\end{array}$ & $\begin{array}{r}52 \\
.000\end{array}$ & $\begin{array}{c}51 \\
0.000 \mathrm{E}\end{array}$ & $\begin{array}{c}50 \\
.000 E+00\end{array}$ & $\stackrel{49}{c .000 E+00}^{4}$ \\
\hline 26 & $\begin{array}{c}58 \\
0.139 \mathrm{E}+02\end{array}$ & $\begin{array}{c}57 \\
.183 E+02\end{array}$ & $\begin{array}{c}56 \\
0.536 E+02\end{array}$ & $\begin{array}{l}55 \\
0.757 E+02\end{array}$ & $\begin{array}{c}54 \\
0.610 E r .02\end{array}$ & $\begin{array}{c}53 \\
0.527 E+01\end{array}$ & $\begin{array}{c}52 \\
0.210 E+01\end{array}$ & $\frac{51}{0.200 E-02}$ & $\begin{array}{r}50 \\
0.000\end{array}$ & $\stackrel{49}{.000 E+00}$ & $\begin{array}{c}48 \\
0.000 E+\infty 00\end{array}$ \\
\hline 25 & $\begin{array}{c}57 \\
0.160 E+\infty 0\end{array}$ & $\begin{array}{c}56 \\
.130 E+01\end{array}$ & $\begin{array}{c}55 \\
.544 E+01\end{array}$ & $\begin{array}{l}54 \\
.969 E+01\end{array}$ & $\begin{array}{c}53 \\
.196 E+02\end{array}$ & $\begin{array}{c}52 \\
0.262 E+02\end{array}$ & $\begin{array}{c}51 \\
.323 E+01\end{array}$ & $\begin{array}{r}50 \\
.170\end{array}$ & $.000 E+00$ & $\frac{48}{.000 E+00}$ & $\frac{47}{0.000 E+00}$ \\
\hline 24 & $\begin{array}{c}56 \\
0.751 E-03\end{array}$ & $\begin{array}{c}55 \\
0.143 E-01\end{array}$ & $\begin{array}{c}54 \\
0.142 E+\infty 0\end{array}$ & $\begin{array}{c}53 \\
0.147 E+01\end{array}$ & $\begin{array}{c}52 \\
.759 E+01\end{array}$ & $\begin{array}{c}51 \\
0.322 E+01\end{array}$ & $\begin{array}{c}50 \\
0.609 \mathrm{E}+\infty 0\end{array}$ & $\begin{array}{c}49 \\
0.934 E+\infty 0\end{array}$ & $\begin{array}{c}48 \\
0.732 E-02\end{array}$ & $\stackrel{47}{0.000 E+00}$ & $0.000 E+\infty$ \\
\hline 23 & $\begin{array}{c}55 \\
.163 E-05\end{array}$ & $\begin{array}{c}54 \\
450 E-06\end{array}$ & $\begin{array}{c}53 \\
.485 E-02\end{array}$ & $\stackrel{52}{0.792 E-01}$ & $\begin{array}{l}51 \\
.104 E+\infty\end{array}$ & $\begin{array}{c}50 \\
0.474 E\end{array}$ & $\begin{array}{c}49 \\
0.105 \mathrm{E}\end{array}$ & $\begin{array}{r}48 \\
.909\end{array}$ & $\stackrel{47}{0.107 E-07}$ & $\frac{46}{0.000 E+00}$ & $\begin{array}{c}45 \\
0.000 E+\infty 0\end{array}$ \\
\hline 22 & $\begin{array}{c}54 \\
0.000 E+\infty 0\end{array}$ & $\begin{array}{c}53 \\
0.000 E+00\end{array}$ & .52 & $\begin{array}{c}51 \\
0.785 E-05\end{array}$ & $\begin{array}{c}50 \\
0.110 E-01\end{array}$ & $\begin{array}{c}49 \\
0.678 E-01\end{array}$ & $\begin{array}{l}48 \\
0.367 E-01\end{array}$ & $0.972 E-02$ & $0.317 E-01$ & $\begin{array}{c}45 \\
0.498 E-03\end{array}$ & $0.000 E+00$ \\
\hline 21 & $\begin{array}{c}53 \\
0.000 E+\infty 0\end{array}$ & $\begin{array}{c}52 \\
0.000 E+00\end{array}$ & $\begin{array}{c}51 \\
.236 E-16\end{array}$ & $\begin{array}{c}50 \\
0.000 E+00\end{array}$ & $\begin{array}{l}49 \\
0.126 \mathrm{E}-03\end{array}$ & $\begin{array}{r}46 \\
.799\end{array}$ & $0.155 \mathrm{E}$ & $\begin{array}{c}46 \\
.345 E\end{array}$ & $\stackrel{45}{537 E-04}$ & $.000 E+00$ & $\frac{43}{0.000 E+00}$ \\
\hline 20 & $\frac{52}{0.000 E+00}$ & $\frac{51}{0.000 E+00}$ & $\begin{array}{l}50 \\
.000 E+\infty 0\end{array}$ & $\frac{49}{0.000 E+\infty}$ & $\begin{array}{c}4 \mathrm{~B} \\
0.221 \mathrm{E}-12\end{array}$ & $.000 E+00$ & 0.101 & 127 & $\begin{array}{c}44 \\
.844 E-04\end{array}$ & $145 E-03$ & $0.145 E-06$ \\
\hline
\end{tabular}

\section{IIId. $100 \mathrm{MeV}$ Yield Cross-Section (MB)}

YIELD CROSS-SECIION (NB)

\begin{tabular}{|c|c|c|c|c|c|c|c|c|c|c|c|}
\hline $\begin{array}{r}71 . A \\
28\end{array}$ & $\begin{array}{c}60 \\
0.000 E+\infty 0\end{array}$ & $\begin{array}{c}59 \\
0.151 E+02\end{array}$ & $\begin{array}{c}58 \\
0.224 E+02\end{array}$ & $\begin{array}{c}57 \\
0.154 E+02\end{array}$ & $\begin{array}{c}56 \\
0.516 E+01\end{array}$ & $\begin{array}{c}55 \\
0.241 E+\infty 0\end{array}$ & $\begin{array}{c}54 \\
0.413 E-02\end{array}$ & $\begin{array}{c}53 \\
0.218 E-0.6\end{array}$ & $0.000 E+\infty 0$ & $\frac{51}{0.000 E+D 0}$ & $\begin{array}{c}50 \\
0.000 E+00\end{array}$ \\
\hline 27 & $\begin{array}{c}59 \\
0.288 E+02\end{array}$ & $\begin{array}{c}58 \\
0.553 E+02\end{array}$ & $\begin{array}{c}57 \\
0.735 E+02\end{array}$ & $\begin{array}{c}56 \\
0.757 E+02\end{array}$ & $\begin{array}{c}55 \\
0.500 E+02\end{array}$ & $\begin{array}{c}54 \\
0.722 E+01\end{array}$ & $\frac{53}{0.277 E+00}$ & $\begin{array}{c}52 \\
0.2^{2} 91 E-04\end{array}$ & $0.000 E+00$ & $\frac{50}{0.000 E+00}$ & $0.000 E+\infty 0$ \\
\hline 26 & $\begin{array}{c}5 B \\
0.144 E+02\end{array}$ & $\begin{array}{c}57 \\
0.150 E+02\end{array}$ & $\begin{array}{c}56 \\
0.469 k+02\end{array}$ & $\begin{array}{c}55 \\
0.767 E+02\end{array}$ & $\begin{array}{c}54 \\
0.754 E+02\end{array}$ & $\begin{array}{c}53 \\
0.184 E+02\end{array}$ & $\begin{array}{c}52 \\
0.999 E+00\end{array}$ & $\stackrel{51}{0.513 \mathrm{E}-01}$ & $\begin{array}{c}50 \\
0.463 E-04\end{array}$ & $0.000 E+00$ & $\begin{array}{c}48 \\
0.000 E+\infty 00\end{array}$ \\
\hline 25 & $\begin{array}{c}57 \\
0.124 E+\infty 0\end{array}$ & $\begin{array}{c}56 \\
0.151 E+01\end{array}$ & $\begin{array}{c}55 \\
0.637 \mathrm{E}+01\end{array}$ & $\frac{54}{0.149 E+02}$ & $\begin{array}{c}53 \\
0.277 \mathrm{E}+02\end{array}$ & $\begin{array}{c}52 \\
0.183 E+02\end{array}$ & $\begin{array}{c}51 \\
0.963 E+01\end{array}$ & $\begin{array}{c}50 \\
0.422 E+00\end{array}$ & $\stackrel{49}{0.665 E-05}$ & $\frac{48}{0.000 E+\infty 0}$ & $\begin{array}{c}47 \\
0.000 E+00\end{array}$ \\
\hline 24 & $\begin{array}{c}56 \\
0.174 E-02\end{array}$ & $\stackrel{55}{0.177 \mathrm{E}-01}$ & $0.262 E+00$ & $\begin{array}{c}53 \\
0.164 E+01\end{array}$ & $\begin{array}{c}52 \\
0.707 E+01\end{array}$ & $\begin{array}{c}51 \\
0.109 E+02\end{array}$ & $\begin{array}{c}50 \\
0.575 E+01\end{array}$ & $\frac{49}{0.519 E+\infty 0}$ & $\frac{48}{0.156 E+\infty 0}$ & $\stackrel{47}{0.106 E-03}$ & $0.000 E+00$ \\
\hline 23 & $\begin{array}{l}55 \\
0.179 E-04\end{array}$ & $0.233 \mathrm{54}-03$ & $\stackrel{53}{0.645 E-02}$ & $\begin{array}{c}52 \\
0.105 E+\infty 0\end{array}$ & $\begin{array}{c}51 \\
0.896 E+\infty 0\end{array}$ & $\begin{array}{c}50 \\
0.809 E+\infty 0\end{array}$ & $\frac{49}{0.803 E+\infty 0}$ & $\begin{array}{c}48 \\
0.131 E+01\end{array}$ & $\frac{47}{0.114 E+\infty}$ & $\begin{array}{c}46 \\
0.401 E-06\end{array}$ & $\frac{45}{0.000 E+\infty 0}$ \\
\hline 22 & $\stackrel{54}{0.419 E-\infty B}$ & $\begin{array}{c}53 \\
0.387 E-\infty 8\end{array}$ & $\begin{array}{c}52 \\
0.552 E-04\end{array}$ & $\frac{51}{0.203 E-02}$ & $\begin{array}{c}50 \\
0.122 \mathrm{E}-01\end{array}$ & $\frac{49}{0.912 \mathrm{E}-01}$ & $\begin{array}{c}48 \\
0.280 E+\infty 0\end{array}$ & $\stackrel{47}{0.158 E+\infty 0}$ & $\frac{46}{0.344 E-01}$ & $\stackrel{45}{0.255 E-01}$ & $0.430 E-03$ \\
\hline 21 & $\begin{array}{c}53 \\
0.000 E+\infty 0\end{array}$ & $0.000 E+\infty$ & $\begin{array}{c}51 \\
0.925 E-07\end{array}$ & $\begin{array}{c}50 \\
0.175 E-06\end{array}$ & $\stackrel{49}{0.109 E-02}$ & $\stackrel{48}{0.505 E-02}$ & $0.194 E-D 2$ & $0.132 E-01$ & $0.237 E-01$ & $0.841 \mathrm{E}-03$ & $\frac{43}{0.701 E-08}$ \\
\hline 20 & $\stackrel{52}{0.000 E+\infty 0}$ & $\stackrel{51}{0.000 E+00}$ & $\begin{array}{c}50 \\
0.758 E-17\end{array}$ & $\stackrel{49}{0.000 E+00}^{49}$ & $\frac{48}{0.470 E-05}$ & $\stackrel{47}{0.113 E-05}^{4}$ & $\begin{array}{c}46 \\
0.192 E-03\end{array}$ & $\begin{array}{c}45 \\
0.915 E-03\end{array}$ & $\frac{44}{0.144 E-03}$ & $\begin{array}{c}43 \\
0.286 E-03\end{array}$ & $\frac{42}{0.114 E-02}$ \\
\hline
\end{tabular}


VIELD CROSS-SECTION (MB)

\begin{tabular}{|c|c|c|c|c|c|c|c|c|c|c|c|}
\hline $\begin{array}{l}21 \mathrm{~A} \\
28\end{array}$ & $0.000 E+00$ & $\begin{array}{c}59 \\
0.118 E+02\end{array}$ & $\begin{array}{c}58 \\
0.165 E+02\end{array}$ & $\begin{array}{c}57 \\
0.120 E+02\end{array}$ & $\begin{array}{c}56 \\
0.307 E+01\end{array}$ & $\stackrel{55}{0.170 E+\infty 0}$ & $\stackrel{54}{0.451 E-02}$ & $\begin{array}{c}53 \\
0.421 E-04\end{array}$ & $\stackrel{52}{0.877 E-\infty 6}$ & $\frac{51}{0.000 E+00}$ & $\stackrel{50}{0.000 E+00}$ \\
\hline 27 & $\stackrel{59}{0.227 E+\infty 2}$ & $\begin{array}{c}58 \\
0.452 E+02\end{array}$ & $\frac{57}{0.620 E+02}$ & $\begin{array}{c}56 \\
0.609 E+02\end{array}$ & $\stackrel{55}{0.350 E+02}$ & $\begin{array}{c}54 \\
0.621 E+01\end{array}$ & $\begin{array}{c}53 \\
0.444 E+\infty\end{array}$ & $\begin{array}{c}52 \\
0.639 E-02\end{array}$ & $\stackrel{51}{0.150 E-\infty}$ & $\begin{array}{c}50 \\
0.000 E+00\end{array}$ & $\frac{49}{0.000 E+00}$ \\
\hline 26 & $\stackrel{58}{0.141 E+02}$ & $\begin{array}{c}57 \\
0.139 E+02\end{array}$ & $\stackrel{56}{0.342 \mathrm{E}+02}$ & $\frac{55}{0.715 E+02}$ & $\begin{array}{c}54 \\
0.734 E+02\end{array}$ & $\frac{53}{0.245 E+02}$ & $\begin{array}{c}52 \\
0.284 E+01\end{array}$ & $\frac{51}{0.540 E-01}$ & 0.12 & $\begin{array}{c}49 \\
0.196 E-05\end{array}$ & 0.48 \\
\hline 25 & $\begin{array}{c}57 \\
0.110 \mathrm{E}+00\end{array}$ & $\begin{array}{c}56 \\
.117 E+01\end{array}$ & $\begin{array}{l}55 \\
739 E+01\end{array}$ & $\begin{array}{c}54 \\
0.151 E+02\end{array}$ & $\begin{array}{c}53 \\
0.371 E+02\end{array}$ & $\begin{array}{r}52 \\
.314\end{array}$ & $\begin{array}{c}51 \\
0.897 \mathrm{E}+01\end{array}$ & $\begin{array}{r}50 \\
0.217\end{array}$ & $0.202 E-01$ & $\begin{array}{c}48 \\
.182 E-05\end{array}$ & $0.000 E+\infty 0$ \\
\hline 24 & $\begin{array}{c}56 \\
0.183 E-02\end{array}$ & $\begin{array}{l}55 \\
0.304 E-01\end{array}$ & $\begin{array}{c}54 \\
.230 E+00\end{array}$ & $\begin{array}{c}53 \\
0.191 E+01\end{array}$ & $\begin{array}{c}52 \\
0.963 E+01\end{array}$ & $\begin{array}{c}51 \\
.108 E+02\end{array}$ & $\begin{array}{c}50 \\
0.129 E+02\end{array}$ & $\begin{array}{c}49 \\
0.383 E+01\end{array}$ & $\begin{array}{l}48 \\
0.176 \mathrm{E}+00\end{array}$ & $\begin{array}{l}47 \\
0.604 E-02\end{array}$ & $0.940 \mathrm{E}-05$ \\
\hline 23 & $\begin{array}{c}55 \\
0.161 E-04\end{array}$ & $\begin{array}{c}54 \\
0.424 E-03\end{array}$ & $\begin{array}{c}53 \\
.127 \mathrm{E}-01\end{array}$ & $\frac{52}{0.150 E+00}$ & $\begin{array}{c}51 \\
0.972 E+\infty 0\end{array}$ & $\begin{array}{c}50 \\
0.292 \mathrm{E}+01\end{array}$ & $0.235 E+01$ & $\stackrel{48}{0.144 E+01}$ & $\begin{array}{c}47 \\
0.862 E+\infty 0\end{array}$ & $\begin{array}{c}46 \\
0.417 E-01\end{array}$ & $0.130 E-05$ \\
\hline 22 & $\begin{array}{c}54 \\
0.154 E-06\end{array}$ & $\begin{array}{c}53 \\
167 \mathrm{E}-05\end{array}$ & $\begin{array}{c}52 \\
0.673 \mathrm{E}-04\end{array}$ & $\begin{array}{c}51 \\
330 E-02\end{array}$ & $\begin{array}{l}50 \\
0.554 E-01\end{array}$ & $\begin{array}{l}49 \\
160 E+00\end{array}$ & $\begin{array}{l}48 \\
.378 E+\infty\end{array}$ & $\begin{array}{c}47 \\
.986 E+00\end{array}$ & $\begin{array}{c}46 \\
0.400 E+\infty 0\end{array}$ & $\begin{array}{l}45 \\
.346 E-01\end{array}$ & $\stackrel{44}{0.188 E-01}$ \\
\hline 21 & $\begin{array}{c}53 \\
0.270 E-10\end{array}$ & $\begin{array}{c}52 \\
.124 E-10\end{array}$ & $\begin{array}{c}51 \\
0.185 E-05\end{array}$ & $\begin{array}{l}50 \\
621 E-04\end{array}$ & $\begin{array}{c}49 \\
0.132 E-02\end{array}$ & $\begin{array}{c}48 \\
0.149 E-01\end{array}$ & $\begin{array}{c}47 \\
427 E-01\end{array}$ & $\begin{array}{r}46 \\
0.241 E\end{array}$ & $\begin{array}{c}45 \\
.418 E\end{array}$ & $\begin{array}{c}44 \\
0.579 E-01\end{array}$ & .833 \\
\hline 20 & $0.138 \mathrm{E}-24$ & $\frac{51}{000 E+00}$ & $\begin{array}{l}50 \\
353 E-09\end{array}$ & 08 & $\begin{array}{r}48 \\
.275\end{array}$ & $\begin{array}{c}47 \\
0.291 E-03\end{array}$ & $\begin{array}{l}46 \\
328 E-03\end{array}$ & 0.27 & $0.970 E \cdot 0 ?$ & $\begin{array}{l}43 \\
389 E-02\end{array}$ & $0.239 \mathrm{E}-02$ \\
\hline
\end{tabular}

Table IIIf. $140 \mathrm{MeV}$ Yield Cross-Section (MB)

VIELO CROSS-SECTION (MB)

\begin{tabular}{|c|c|c|c|c|c|c|c|c|c|c|c|}
\hline $\begin{array}{l}21 A \\
28\end{array}$ & $0.000 E+00$ & $\begin{array}{c}59 \\
0.935 E+01\end{array}$ & $\begin{array}{c}58 \\
0.121 E+02\end{array}$ & $\begin{array}{c}57 \\
0.948 E+01\end{array}$ & $\begin{array}{c}56 \\
0.273 E+01\end{array}$ & $\begin{array}{c}55 \\
0.111 E+00\end{array}$ & $\begin{array}{c}54 \\
0.383 \mathrm{E}-02\end{array}$ & $\begin{array}{l}53 \\
0.731 E-04\end{array}$ & $0.116 E-05$ & $0.321 E-09$ & $\stackrel{50}{0.152 \mathrm{E}-19}$ \\
\hline 27 & $\stackrel{59}{0.180 E+02}$ & $\begin{array}{c}58 \\
0.391 E+02\end{array}$ & $0.533 E+02$ & $\begin{array}{c}55 \\
0.488 E+02\end{array}$ & $\begin{array}{c}55 \\
0.279 \mathrm{E}+02\end{array}$ & $\begin{array}{c}54 \\
0.577 \mathrm{E}+01\end{array}$ & $\stackrel{53}{0.344 E+\infty}$ & $\begin{array}{c}5 z \\
0.204 E-01\end{array}$ & $\frac{51}{0.101 E-03}$ & $\stackrel{50}{0.143 E-06}$ & $0.000 E+C$ \\
\hline 26 & $\begin{array}{c}58 \\
0.137 E+02\end{array}$ & $\begin{array}{c}57 \\
0.140 E+02\end{array}$ & $\begin{array}{c}56 \\
0.299 E+02\end{array}$ & $\begin{array}{c}55 \\
0.509 E+0 ?\end{array}$ & $0.818 \mathrm{E}+02$ & $\begin{array}{c}53 \\
0.220 E+02\end{array}$ & $\begin{array}{c}52 \\
0.453 E+01\end{array}$ & $\begin{array}{c}51 \\
0.140 E+00\end{array}$ & $\stackrel{50}{0.140 E-02}$ & $\begin{array}{c}49 \\
0.534 E-04\end{array}$ & -07 \\
\hline 25 & $\begin{array}{c}57 \\
0.109 \mathrm{E}+\infty 0\end{array}$ & $\begin{array}{c}56 \\
0.991 E+09\end{array}$ & $\begin{array}{c}55 \\
0.658 E+01\end{array}$ & 0.174 & $\begin{array}{c}53 \\
0.348 E+02\end{array}$ & $0.340 E+02$ & $\begin{array}{l}51 \\
.143 E+02\end{array}$ & 01 & -01 & $\begin{array}{l}48 \\
.659 E-03\end{array}$ & $\begin{array}{r}47 \\
0.610\end{array}$ \\
\hline 24 & $\begin{array}{c}56 \\
0.133 \mathrm{E}-02\end{array}$ & $\begin{array}{c}55 \\
0.312 E-01\end{array}$ & $\begin{array}{c}54 \\
0.298 E+\infty\end{array}$ & $\stackrel{53}{0.163 E+01}$ & $\begin{array}{c}52 \\
0.102 E+02\end{array}$ & $\begin{array}{l}51 \\
0.154 E+02\end{array}$ & $\begin{array}{c}50 \\
0.132 E+02\end{array}$ & $0.872 E+01$ & $\begin{array}{c}48 \\
0.741 E+\infty 0\end{array}$ & 0.97 & $\begin{array}{c}46 \\
0.399 E-03\end{array}$ \\
\hline 23 & $\stackrel{55}{0.331 E-04}$ & $\begin{array}{c}54 \\
.378 E-03\end{array}$ & $\stackrel{53}{0.321 E-01}$ & $\frac{52}{0.206 E+00}$ & $\begin{array}{c}51 \\
0.126 E+01\end{array}$ & $\frac{50}{0.2 B 5 E+01}$ & $\frac{49}{0.494 E+01}$ & $\frac{48}{0.442 E+01}$ & $\stackrel{47}{0.102 E+01}$ & $\begin{array}{c}46 \\
0.301 E+\infty C\end{array}$ & $\begin{array}{c}45 \\
0.247 E-02\end{array}$ \\
\hline 22 & $\begin{array}{c}54 \\
0.173 E-06\end{array}$ & $\begin{array}{c}53 \\
0.377 E-05\end{array}$ & $\frac{52}{0.155 \varepsilon-03}$ & $\begin{array}{c}51 \\
0.323 E-02\end{array}$ & $\begin{array}{c}50 \\
0.704 \mathrm{E}-01\end{array}$ & $\begin{array}{c}49 \\
0.38 B E+\infty\end{array}$ & $\begin{array}{c}48 \\
0.98 B E+\infty\end{array}$ & $\begin{array}{c}47 \\
0.866 \mathrm{E}+00\end{array}$ & $\frac{46}{0.161 E+01}$ & $\begin{array}{c}45 \\
0.465 E+\infty 0\end{array}$ & $\stackrel{44}{0.299 E-01}^{2}$ \\
\hline 21 & $\begin{array}{l}53 \\
951 E-09\end{array}$ & $\begin{array}{c}52 \\
0.857 E-08\end{array}$ & $\stackrel{51}{.209 E-C 5}$ & $\begin{array}{c}50 \\
0.148 E-03\end{array}$ & $\stackrel{49}{403 E-02}$ & $\begin{array}{c}48 \\
0.152 E\end{array}$ & $\begin{array}{c}47 \\
0.713 E-01\end{array}$ & $160 E+00$ & $\begin{array}{c}45 \\
0.144 E+00\end{array}$ & $\begin{array}{c}44 \\
0.102 E\end{array}$ & $\begin{array}{c}43 \\
.735 E-01\end{array}$ \\
\hline 20 & $\frac{52}{.222 E-13}$ & $\begin{array}{l}51 \\
250 E-13\end{array}$ & $\begin{array}{l}50 \\
.718 E-\infty 8\end{array}$ & $\begin{array}{c}49 \\
0.399 E-\infty 6\end{array}$ & .48 & $\begin{array}{r}47 \\
0.730\end{array}$ & $0.343 \mathrm{E}-02$ & $\begin{array}{l}45 \\
491 E-02\end{array}$ & $0.196 \mathrm{E}-01$ & $\begin{array}{c}43 \\
0.430 E-01\end{array}$ & $\begin{array}{c}42 \\
0.308 E-01\end{array}$ \\
\hline
\end{tabular}




\section{IIIg. $160 \mathrm{MeV}$ Yield Cross-Section (MB)}

Y[ELO CROSS-SECTION (ME)

\begin{tabular}{|c|c|c|c|c|c|c|c|c|c|c|c|}
\hline $\begin{array}{l}214 \\
28\end{array}$ & $0.000 E+\infty$ & $\begin{array}{c}59 \\
0.906 E+01\end{array}$ & $\begin{array}{c}58 \\
0.100 E+02\end{array}$ & $\begin{array}{c}57 \\
0.742 E+01\end{array}$ & $\begin{array}{c}56 \\
0.222 E+01\end{array}$ & $\begin{array}{c}55 \\
0.879 E-01\end{array}$ & $\begin{array}{c}54 \\
0.256 \mathrm{E}-02\end{array}$ & $\begin{array}{c}53 \\
0.441 E-04\end{array}$ & $\begin{array}{c}52 \\
0.169 E-05\end{array}$ & $\begin{array}{c}51 \\
0.183 E-07\end{array}$ & $\begin{array}{c}50 \\
0.475 E-12\end{array}$ \\
\hline 27 & $\stackrel{59}{0.17] \mathrm{E}+02}$ & $\begin{array}{c}58 \\
0.342 E+02\end{array}$ & $\begin{array}{c}57 \\
.469 \mathrm{E}+02\end{array}$ & $\begin{array}{c}56 \\
0.404 E+02\end{array}$ & $\begin{array}{c}55 \\
0.235 E+02\end{array}$ & $\begin{array}{c}54 \\
0.556 E+01\end{array}$ & $\begin{array}{r}5: \\
0,484 E\end{array}$ & $\begin{array}{c}52 \\
0.206 \mathrm{E}\end{array}$ & $\begin{array}{c}51 \\
0.422 E\end{array}$ & $\begin{array}{c}50 \\
0.16 \& E-05\end{array}$ & 49 \\
\hline 26 & $\begin{array}{c}58 \\
0.128 E+02\end{array}$ & $\stackrel{57}{0.124 E+02}$ & $\begin{array}{c}56 \\
.296 E+02\end{array}$ & $\begin{array}{l}55 \\
.446 E+02\end{array}$ & $\begin{array}{c}54 \\
0.696 \mathrm{E}+02\end{array}$ & $0.2^{53}$ & $\begin{array}{c}52 \\
0.326 E+01\end{array}$ & $0.255 E+\infty$ & $0.48^{5}$ & $\begin{array}{c}49 \\
0.109 \mathrm{E}-03\end{array}$ & $\begin{array}{c}48 \\
0.509 E-\end{array}$ \\
\hline 25 & $\frac{57}{0.958 E-01}$ & $\begin{array}{l}56 \\
.955 E+00\end{array}$ & $\begin{array}{c}55 \\
0.57 \mathrm{AE}+01\end{array}$ & $\begin{array}{l}54 \\
.181 E+02\end{array}$ & $\begin{array}{c}53 \\
0.367 \mathrm{E}+02\end{array}$ & $0.292 \mathrm{E}+02$ & $\begin{array}{c}51 \\
0.163 E+02\end{array}$ & $\begin{array}{l}50 \\
.288 E+01\end{array}$ & & 0.36 & $0.153 \mathrm{E}-04$ \\
\hline 24 & $\begin{array}{l}56 \\
.154 E-02\end{array}$ & $\begin{array}{c}55 \\
.310 E-01\end{array}$ & $\begin{array}{c}54 \\
0.375 E+00\end{array}$ & $\begin{array}{c}53 \\
.180 E+01\end{array}$ & $\stackrel{52}{0.915 E+01}$ & $\frac{51}{0.175 E+02}$ & $\begin{array}{c}50 \\
0.200 E+02\end{array}$ & $\begin{array}{c}49 \\
0.739 E+01\end{array}$ & $\begin{array}{c}48 \\
0.255 E+01\end{array}$ & $0.304 \mathrm{E}-01$ & $0.664 \mathrm{E}-0 \mathrm{~J}$ \\
\hline 23 & $\begin{array}{l}55 \\
478 E-04\end{array}$ & $\begin{array}{l}54 \\
0.726 E-03\end{array}$ & $\begin{array}{c}53 \\
0.107 \mathrm{E}-01\end{array}$ & $\begin{array}{c}52 \\
.192 E+C 0\end{array}$ & $\begin{array}{c}51 \\
0.176 E+01\end{array}$ & $\begin{array}{c}50 \\
0.353 E+01\end{array}$ & .49 & $\begin{array}{r}48 \\
0.700 E\end{array}$ & $\frac{d 7}{0.229 E+01}$ & $\begin{array}{c}46 \\
0.328 E+\infty 0\end{array}$ & $0.853 \mathrm{E}-02$ \\
\hline 22 & $\begin{array}{l}54 \\
116 E-06\end{array}$ & $\begin{array}{c}53 \\
0.269 E-05\end{array}$ & $\begin{array}{l}52 \\
190 E-03\end{array}$ & $\begin{array}{c}51 \\
562 E-02\end{array}$ & $\begin{array}{c}50 \\
67 \mathrm{gE}-01\end{array}$ & $\begin{array}{c}49 \\
0.405 E+00\end{array}$ & $\stackrel{48}{.119 E+01}$ & $\stackrel{47}{.216 E+01}^{4}$ & $\stackrel{45}{0.199 E+01}$ & $\begin{array}{c}45 \\
0.102 E+01\end{array}$ & $0.133 E+\infty$ \\
\hline 21 & $\begin{array}{c}53 \\
277 \mathrm{E}-08\end{array}$ & $\begin{array}{c}52 \\
0.417 E-07\end{array}$ & $\begin{array}{c}51 \\
.235 E-05\end{array}$ & $\begin{array}{c}50 \\
0.163 \mathrm{E}-03\end{array}$ & $\begin{array}{l}49 \\
.679 E-02\end{array}$ & $\begin{array}{c}48 \\
0.381 E-01\end{array}$ & $\begin{array}{c}47 \\
0.940 E-01\end{array}$ & $\begin{array}{c}46 \\
.226 E+00\end{array}$ & $\begin{array}{c}45 \\
0.460 E+\infty 0\end{array}$ & $.257 E+00$ & $\begin{array}{c}43 \\
0.927 E-01\end{array}$ \\
\hline & $.159 \mathrm{E}-11$ & $\stackrel{51}{155 E-10}$ & $\begin{array}{l}50 \\
.110 E-07\end{array}$ & $\begin{array}{l}49 \\
109 E-05\end{array}$ & $\stackrel{48}{406 E-03}$ & $0.974 E-03$ & $0.681 E-02$ & $.211 \mathrm{E}-01$ & $0.388 \mathrm{E}-01$ & $.564 E-01$ & $0.127 E+\infty 0$ \\
\hline
\end{tabular}

IIIh. $180 \mathrm{MeV}$ Vield Cross-Section (MB)

YIELD CROSS-SECTION (MB)

\begin{tabular}{|c|c|c|c|c|c|c|c|c|c|c|c|}
\hline $\begin{array}{r}Z 1 A \\
2 B\end{array}$ & $\begin{array}{c}60 \\
0.000 E+00\end{array}$ & $\begin{array}{c}59 \\
0.744 E+01\end{array}$ & $\begin{array}{c}58 \\
0.829 E+01\end{array}$ & $\begin{array}{c}57 \\
0.632 E+01\end{array}$ & $\begin{array}{c}56 \\
0.154 E+01\end{array}$ & $\begin{array}{c}55 \\
0.702 \mathrm{E}-01\end{array}$ & $\begin{array}{c}54 \\
0.266 \mathrm{E}-02\end{array}$ & $\begin{array}{c}53 \\
0.558 \mathrm{E}-04\end{array}$ & $0.153 \mathrm{E}-05$ & $\begin{array}{c}51 \\
0.780 E-07\end{array}$ & $\begin{array}{c}50 \\
0.106 E-09\end{array}$ \\
\hline 27 & $\begin{array}{c}59 \\
0.141 E+02\end{array}$ & $\begin{array}{c}58 \\
0.293 E+02\end{array}$ & $\begin{array}{c}57 \\
0.414 E+02\end{array}$ & $\begin{array}{c}56 \\
0.373 E+02\end{array}$ & $\begin{array}{c}55 \\
0.182 E+\infty 2\end{array}$ & $\begin{array}{c}54 \\
0.460 E+01\end{array}$ & $\begin{array}{c}53 \\
0.469 E+00\end{array}$ & $\begin{array}{c}52 \\
0.187 E-01\end{array}$ & $\stackrel{51}{0.531 E-\infty]}$ & $\begin{array}{c}50 \\
0.774 E-05\end{array}$ & $\begin{array}{l}49 \\
0.630 \mathrm{E}-08\end{array}$ \\
\hline 26 & $\begin{array}{c}58 \\
0.117 \mathrm{E}+102\end{array}$ & $\begin{array}{c}57 \\
0.116 E+02\end{array}$ & $\begin{array}{c}56 \\
0.23 \mathrm{BE}+02\end{array}$ & $\begin{array}{c}55 \\
0.413 E+02\end{array}$ & $\begin{array}{c}54 \\
0.563 E+02\end{array}$ & $\begin{array}{c}53 \\
0.294 E+02\end{array}$ & $\begin{array}{c}52 \\
0.367 E+01\end{array}$ & $\begin{array}{c}51 \\
0.239 E+\infty 0\end{array}$ & $\begin{array}{c}50 \\
0.860 E-02\end{array}$ & $\begin{array}{c}49 \\
0.174 E-03\end{array}$ & $\begin{array}{c}48 \\
0.225 E-05\end{array}$ \\
\hline 25 & $\begin{array}{c}57 \\
0.892 E-01\end{array}$ & $\begin{array}{c}56 \\
0.809 \mathrm{E}+00\end{array}$ & $\begin{array}{c}55 \\
0.483 E+01\end{array}$ & $\begin{array}{c}54 \\
0.158 E+02\end{array}$ & $\begin{array}{c}53 \\
0.459 E+02\end{array}$ & $\begin{array}{c}52 \\
0.329 E+02\end{array}$ & $\begin{array}{c}51 \\
0.143 E+02\end{array}$ & $\begin{array}{c}50 \\
0.426 E+01\end{array}$ & $\begin{array}{c}49 \\
0.880 E-01\end{array}$ & $\begin{array}{c}48 \\
0.479 \mathrm{E}-02\end{array}$ & $\stackrel{47}{0.863 E-04}$ \\
\hline 24 & $\begin{array}{c}56 \\
0.122 E-02\end{array}$ & $\begin{array}{c}55 \\
0.255 E-01\end{array}$ & $\begin{array}{c}54 \\
0.349 E+00\end{array}$ & $\frac{53}{0.220 E+01}$ & $\stackrel{52}{0.900 E+01}$ & $\begin{array}{c}51 \\
0.166 E+02\end{array}$ & $\begin{array}{c}50 \\
0.234 E+02\end{array}$ & $\begin{array}{c}49 \\
0.106 E+02\end{array}$ & $\begin{array}{c}48 \\
0.187 E+01\end{array}$ & $\begin{array}{c}47 \\
0.806 E-01\end{array}$ & $\begin{array}{c}46 \\
0.162 E-02\end{array}$ \\
\hline 23 & $\begin{array}{c}55 \\
0.446 \mathrm{E}-04\end{array}$ & $\begin{array}{c}54 \\
0.102 E-02\end{array}$ & $\begin{array}{c}53 \\
0.143 E-01\end{array}$ & $\begin{array}{l}52 \\
0.172 E+C 0\end{array}$ & $\begin{array}{c}51 \\
0.183 E+01\end{array}$ & $\begin{array}{c}50 \\
0.511 E+01\end{array}$ & $\stackrel{49}{0.566 E+01}$ & $\frac{48}{0.755 E+01}$ & $\begin{array}{c}47 \\
0.405 E+01\end{array}$ & $\begin{array}{c}46 \\
0.636 E+00\end{array}$ & $\begin{array}{c}45 \\
0.124 \mathrm{E}-01\end{array}$ \\
\hline 22 & $\stackrel{54}{0.304 E-06}$ & $\stackrel{53}{0.385 E-05}$ & $\frac{52}{0.219 E-03}$ & $\frac{51}{0.775 E-02}$ & $\begin{array}{c}50 \\
0.100 E+\infty 0\end{array}$ & $\begin{array}{c}49 \\
0.413 E+\infty 0\end{array}$ & $\frac{48}{0.142 E+01}$ & $\frac{47}{0.366 E+01}$ & $\frac{46}{0.330 E+01}$ & $\begin{array}{c}45 \\
0.141 E+01\end{array}$ & $\stackrel{44}{0.546 E+\infty 0}$ \\
\hline 21 & $\frac{53}{0.280 E-08}$ & $\begin{array}{c}52 \\
0.556 E-07\end{array}$ & $\stackrel{51}{0.546 \mathrm{E}-05}$ & $\stackrel{50}{0.195 E-03}$ & $\begin{array}{l}49 \\
0.777 E-02\end{array}$ & $\begin{array}{c}48 \\
0.577 E-01\end{array}$ & $\begin{array}{c}47 \\
0.176 E+\infty\end{array}$ & $0.296 E+00$ & $\begin{array}{c}45 \\
0.621 E+00\end{array}$ & $\stackrel{44}{0.795 E+00}$ & $\stackrel{43}{0.334 E+\infty}$ \\
\hline 20 & $\stackrel{52}{0.103 E-10}$ & $\begin{array}{c}51 \\
0.137 \mathrm{E}-09\end{array}$ & $\begin{array}{c}50 \\
0.191 E-07\end{array}$ & $\begin{array}{c}49 \\
0.125 E-05\end{array}$ & $\begin{array}{c}48 \\
0.170 E-03\end{array}$ & $\begin{array}{c}47 \\
0.182 E-02\end{array}$ & $\frac{46}{0.820 E-0 E}$ & $\begin{array}{c}45 \\
0.325 E-01\end{array}$ & $0.108 E+\infty$ & $\begin{array}{c}43 \\
0.140 E+00\end{array}$ & $0.208 E+\infty 0$ \\
\hline
\end{tabular}


YIELO CROSS-SECTION (ME)

\begin{tabular}{|c|c|c|c|c|c|c|c|c|c|c|c|}
\hline Z1A & $\begin{array}{c}60 \\
0.000 E+\infty 0\end{array}$ & $\begin{array}{c}59 \\
0.614 E+01\end{array}$ & $\begin{array}{c}58 \\
0.658 E+01\end{array}$ & $\begin{array}{c}57 \\
0.548 E+01\end{array}$ & $\begin{array}{c}56 \\
0.153 E+01\end{array}$ & $\begin{array}{l}55 \\
.537 E-D 1\end{array}$ & $\begin{array}{c}54 \\
0.202 \mathrm{E}-02\end{array}$ & $\begin{array}{c}53 \\
0.596 \mathrm{E}-04\end{array}$ & $\begin{array}{c}52 \\
0.136 E-05\end{array}$ & $\frac{51}{0.950 E-07}$ & $\begin{array}{c}50 \\
0.610 \mathrm{E}-09\end{array}$ \\
\hline 27 & $\begin{array}{c}59 \\
0.117 E+02\end{array}$ & $\begin{array}{c}58 \\
0.257 E+02\end{array}$ & $\frac{57}{0.369 F+02}$ & $\begin{array}{c}56 \\
.320 F+02\end{array}$ & $\begin{array}{c}55 \\
0.163 E\end{array}$ & $\begin{array}{c}54 \\
.353 E\end{array}$ & $\begin{array}{c}53 \\
.420 E+\infty 0\end{array}$ & $\begin{array}{r}52 \\
0.204\end{array}$ & 0.3 & 24 & 19 \\
\hline 26 & $\begin{array}{l}58 \\
112 E+02\end{array}$ & $\begin{array}{c}57 \\
.112 E\end{array}$ & $\begin{array}{c}56 \\
0.218 E+02\end{array}$ & $\begin{array}{r}55 \\
.318\end{array}$ & $\begin{array}{c}54 \\
.519 E+02\end{array}$ & $\begin{array}{r}53 \\
.273\end{array}$ & $\begin{array}{r}52 \\
.530\end{array}$ & $\begin{array}{r}51 \\
191\end{array}$ & 02 & 3 & 05 \\
\hline 25 & $\begin{array}{c}57 \\
0.901 E-01\end{array}$ & $\begin{array}{l}56 \\
.719 E+O U\end{array}$ & $\begin{array}{c}55 \\
0.421 E+01\end{array}$ & $\begin{array}{c}54 \\
0.135 E+02\end{array}$ & $\stackrel{53}{.423 E+02}$ & $\begin{array}{c}52 \\
0.374 E+02\end{array}$ & $\stackrel{51}{0.132 E+02}$ & $\begin{array}{r}5 \\
0.42\end{array}$ & $\begin{array}{c}49 \\
0.114 E+\infty\end{array}$ & $\begin{array}{r}48 \\
0.531\end{array}$ & $\begin{array}{r}47 \\
0.125\end{array}$ \\
\hline 24 & $\begin{array}{l}56 \\
953 E-03\end{array}$ & $211 E-01$ & $0.304 E+\infty 0$ & $\begin{array}{c}53 \\
0.213 E+01\end{array}$ & $\stackrel{52}{.996 E+01}$ & $\stackrel{51}{0.145 E+02}$ & $\begin{array}{c}50 \\
0.243 E+02\end{array}$ & $\begin{array}{r}49 \\
.157\end{array}$ & $\begin{array}{c}48 \\
0.190 E+01\end{array}$ & 0.106 & $0.531 E-02$ \\
\hline 23 & $\begin{array}{l}55 \\
464 E-04\end{array}$ & $\begin{array}{c}54 \\
0.986 \mathrm{E}-03\end{array}$ & $\begin{array}{c}53 \\
0.171 E-01\end{array}$ & $\begin{array}{c}52 \\
0.178 E+00\end{array}$ & $\begin{array}{c}51 \\
166 E+01\end{array}$ & $\begin{array}{r}50 \\
.569\end{array}$ & $\begin{array}{c}49 \\
0.789 \mathrm{E}\end{array}$ & $\begin{array}{c}48 \\
.907 E+01\end{array}$ & $\begin{array}{c}47 \\
0.482 E+01\end{array}$ & $\begin{array}{r}46 \\
0.130\end{array}$ & $\begin{array}{r}45 \\
.263\end{array}$ \\
\hline 22 & $\frac{54}{454 E-06}$ & $\begin{array}{c}53 \\
0.662 E-05\end{array}$ & $\begin{array}{c}52 \\
.184 E-03\end{array}$ & $\begin{array}{l}51 \\
0.710 E-02\end{array}$ & $\begin{array}{l}50 \\
140 E+00\end{array}$ & $\begin{array}{c}49 \\
0.552 E+00\end{array}$ & $\begin{array}{c}48 \\
0.195 E+01\end{array}$ & $0.326 E+01$ & $\begin{array}{c}46 \\
.569 t+01\end{array}$ & 0.262 & $0.652 E+\infty$ \\
\hline 21 & $193 \mathrm{E}-$ & $0.563 E-07$ & $726 \mathrm{E}-$ & $\begin{array}{r}50 \\
0.269 \mathrm{E}\end{array}$ & $\begin{array}{r}49 \\
763\end{array}$ & $\begin{array}{r}48 \\
0.5528\end{array}$ & $\begin{array}{r}47 \\
0.231\end{array}$ & $\begin{array}{r}46 \\
.479 E\end{array}$ & $\begin{array}{c}45 \\
0.847 E\end{array}$ & $\begin{array}{r}44 \\
.115\end{array}$ & $\frac{43}{0.755 E \cdot 00}$ \\
\hline 3 & $.102 \mathrm{E}-10$ & $\begin{array}{c}51 \\
0.223 \mathrm{E}-09\end{array}$ & $\begin{array}{c}50 \\
0.205 E-07\end{array}$ & $\stackrel{49}{0.132 E-05}$ & $\begin{array}{l}48 \\
190 E-03\end{array}$ & $\begin{array}{c}47 \\
.264 E-\end{array}$ & $\begin{array}{r}46 \\
0.133\end{array}$ & $394 E-01$ & $.151 E+\infty 0$ & $0.310 E+\infty 0$ & $0.447 \mathrm{E}+00$ \\
\hline
\end{tabular}

Table IV. ALICE Parameter Variation for Benchmark 2

Case MP $\underline{\text { INVER }} \underline{M 3} \quad \underline{\text { Notes }}$

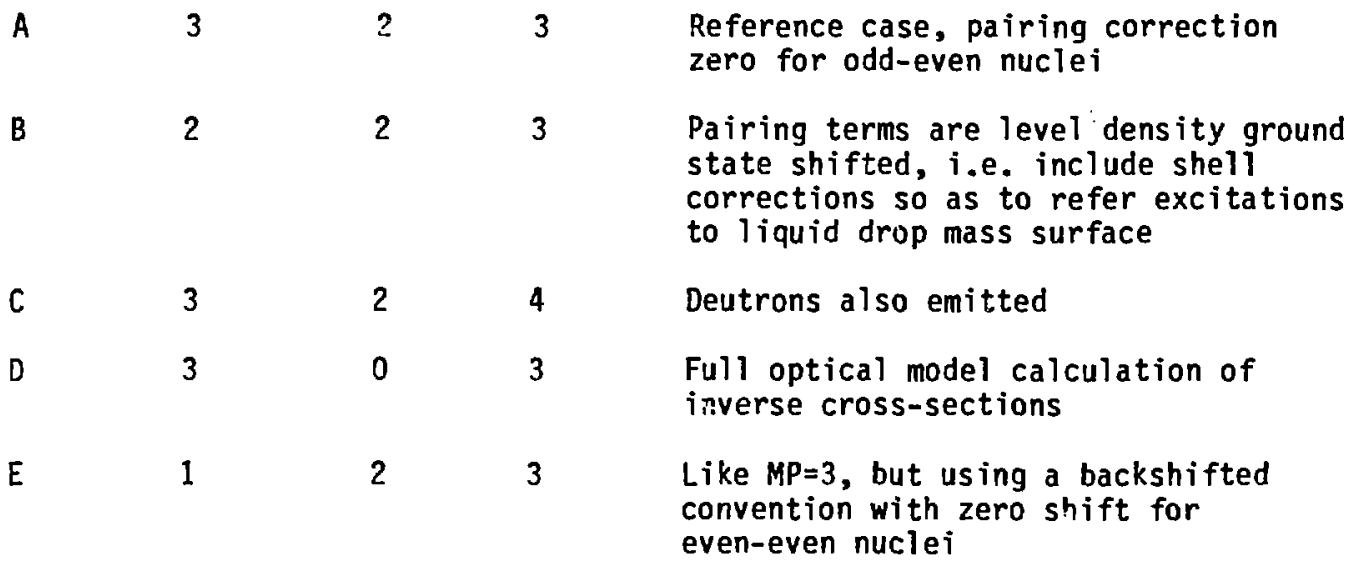


The benchmark specifications called for results binned in certain secondary angular and energy increments. The results received did not necessarily conform to the specifications. This made tabular comparisons difficult but the results were sufficiently detailed to provide meaningful graphical comparisons.

Data for all three targets $A L-27, C_{0-59}$, and $P B-208$ were received from submitters. Additional details are given in Table $V$.

For ALICE results, the point symbols are plotted at the center of each bin. For HETC-F results, the integrals between energy points on a nonequidistant energy mesh were given and the point symbols are plotted at the arithmetic midpoint of the bins. For HETC-H results, cross-section point symbols are plotted on the energy mesh given, which is assumed to be the bin midpoints. For improved readability, histogran curves were not drawn through these data.

The PNEM results were generated on a comparatively fine mesh with a curve connecting the points without symbols.

As an example of the changes that occurred from the draft report, Figure 4 shows the calculated $800 \mathrm{MeV}$ proton induced neutron emission spectrum at $30^{\circ}$. The HETC-H results with corrected normalization are higher by factors of 1 to 3 than the draft report.

A log-linear plot of the per unit lethargy cross-section as shown in Figure 5 is useful for displaying details in neutron emission spectra thoughout the energy range. However, the log-log display used in this section is sufficient to globally and compactly compare how well the codes agree without requiring a separate figure for each target and input energy or numerous changes in scale.

While emission spectra can be compared at 10 angles, only selected graphical comparisons will be given. The information has been saved and additional tabular and graphical comparisons can be given at a later time if warranted.

Neutron emission spectra at $15,30,60,90,120$, and 150 degrees are shown in Figures 6, 7, 8, 9, and 10 for 35, 80, 160, 318, and $800 \mathrm{MeV}$, respectively. The ALICE results at 35,80 , and $160 \mathrm{MeV}$ are the computation format results from ALICE-B obtained with the same input parameters selected by $M$. Blann. The results have been compared with those of ALICE and are equivalent, except for the small differences indicated earlier. While ALICE-B results for 318 and $800 \mathrm{MeV}$ have been submitted by S. Pearlstein it does not imply that the authors of ALICE, from which ALICE-B was derived, have suggested the use of its algorithms beyond $300 \mathrm{MeV}$. 


\section{Table V. Mesh Sizes of Contributed Benchmarks}

\begin{tabular}{|c|c|c|c|}
\hline Code & Energy & $\begin{array}{c}\text { Angle } \\
\text { Bin (Deg), Mesh Pts, }\end{array}$ & $\begin{array}{c}\text { Energy } \\
\text { Bin (MeV), Mesh Pts. }\end{array}$ \\
\hline ALICE $^{1}$ & $\begin{array}{r}35 \\
80 \\
160\end{array}$ & $\begin{array}{l}5,36 \\
5,36 \\
5,36\end{array}$ & $\begin{array}{l}1,5 \\
1,7 \\
1,14\end{array}$ \\
\hline ALICE-B ${ }^{2}$ & $\begin{array}{l}318 \\
800\end{array}$ & $\begin{array}{l}5,36 \\
5,36\end{array}$ & $\begin{array}{l}5,64 \\
5,160\end{array}$ \\
\hline HETC-F ${ }^{3}$ & $\begin{array}{r}35 \\
80 \\
160 \\
318 \\
800\end{array}$ & $\begin{array}{ll}3-10, & 10 \\
3-10, & 10 \\
3-10, & 10 \\
3-10, & 10 \\
3-10, & 10\end{array}$ & $\begin{array}{c}2,12-16 \\
5,12-16 \\
15,5,11-13 \\
2.6-118,11-17 \\
3.3-328,11-17\end{array}$ \\
\hline HETC $-H^{4}$ & $\begin{array}{l}160 \\
318 \\
800\end{array}$ & $\begin{array}{l}5,36 \\
3,60 \\
3,60\end{array}$ & $\begin{array}{ll}10,16 \\
20,17 \\
50,16\end{array}$ \\
\hline PNEM & All & Continuous & Continuous, $19 /$ decade \\
\hline
\end{tabular}

NOTES

1. Other $A L I C E$ parameters are $N A=9, N Z=5, M P=1$, INVER $=2, M 3=0$, and $I 3 D=0$ ( 3 dimensional folding). For computerized output, ALICE-B results were used with these parameters.

2. In ALICE-B, ALICE algorithms are extended to $1000 \mathrm{MeV}, P$ values to 70 and the number of secondary energy bins increased for double differential distributions.

3. Results were received at emission angles of $3,15,30,45,60$, $75,90,105,120,150$ degrees. The low energy cut off is 5 MeV.

4. The number of energy bins and bin sizes received differ somewhat from those stated in Appendix II.5.

The angular integrated results and the angular and energy integrated totals, the $(p, x n)$ cross-section, are also of interest. The angular integrated results are shown in Figure 11. The energy-angle integrated totals are listed in Table VI. Figures 6 through 11 can serve as a shape comparisons and Table $V I$ as a magnitude comparison for others calculating these benchmarks. 


\section{III.3 Calculation Times}

Some information on calculation times was received. From $M$. Blann's ALICE output from the COC-7600, a 3.3 MIPS computer, the CPU times for Benchmarks 1 and 2, 1.6 minutes, and at 35, 80, and $160 \mathrm{MeV}$, for Benchmarks 4 and $5,2.8$ minutes.

PNEM calculation times on the DEC-10, a 0.3 MIPS computer, were 0.8 minutes for each of the Benchmarks 3,4 , or 5 .

For the HETC-H calculations received at 160,318 , and $800 \mathrm{MeV}$ for Benchmarks 3, 4, and 5, the timing information is shown in Table VII. The calculations were run on an IBM 3081 , a 2.2 MIPS computer. The average benchmark time was 6.5 hours.

Table VI. The $(p, x n)$ Cross-Sections $(m b)$

\begin{tabular}{|c|c|c|c|c|c|c|c|}
\hline \multirow[b]{2}{*}{ Target } & \multirow[b]{2}{*}{ Energy } & \multirow[b]{2}{*}{ PNEM } & \multirow[b]{2}{*}{ ALICE } & \multirow[b]{2}{*}{ HETC-F* } & \multirow[b]{2}{*}{ HETC-H } & \multicolumn{2}{|c|}{ Unweighted } \\
\hline & & & & & & Av. & $\underline{\text { Unc }}$ \\
\hline A1 & 35 & 260 & 460 & 240 & & 360 & \\
\hline & 80 & 450 & 600 & 350 & & 530 & $\begin{array}{l}5 \\
?\end{array}$ \\
\hline & 160 & 710 & 600 & 390 & 660 & 660 & 2 \\
\hline & 318 & 1100 & 990 & 460 & 530 & 870 & 18 \\
\hline & 800 & 1800 & 1500 & 1000 & 590 & 1300 & 3 \\
\hline $\mathrm{Zr}$ & 35 & 1200 & 2200 & 440 & & 1700 & \\
\hline & 80 & 2200 & 2900 & 790 & & 2500 & \\
\hline & 160 & 3400 & 3100 & 1100 & 3000 & 3200 & 12 \\
\hline & 318 & 5200 & 4200 & 1600 & 1900 & 3800 & \\
\hline & 800 & 8800 & 5400 & 3600 & 1500 & 5200 & 210 \\
\hline $\mathrm{Pb}$ & 35 & 2800 & 6100 & 930 & & 4500 & 1 \\
\hline & 80 & 4800 & 10000 & 1500 & & 7400 & 36 \\
\hline & 160 & 7300 & 11000 & 2300 & 9800 & 9400 & 110 \\
\hline & 318 & 14000 & 12000 & 3700 & 4700 & 10000 & 28 \\
\hline & 800 & 25000 & 15000 & 8700 & 2800 & 14000 & \\
\hline
\end{tabular}

*The energy bins do not extend down to zero energy. This column was not used in the unweighted averages. 
Table VII. HETC-H Run Characteristics

\begin{tabular}{|c|c|c|c|}
\hline $\begin{array}{c}\text { Proton Energy } \\
(\mathrm{MeV}) \\
\end{array}$ & Target Material & $\begin{array}{c}\text { Number of } \\
\text { Source Particles } \\
\end{array}$ & $\begin{array}{ll}\text { Run Time } & \text { On } \\
\text { IBM } 3081 & \text { (h) } \\
\end{array}$ \\
\hline $\begin{array}{l}800 \\
316 \\
160\end{array}$ & $\begin{array}{l}\mathrm{Pb}-208 \\
\mathrm{~Pb}-208 \\
\mathrm{~Pb}-208\end{array}$ & $\begin{array}{l}240000 \\
280000 \\
520000\end{array}$ & $\begin{array}{l}6.1 \\
6.9 \\
6.4\end{array}$ \\
\hline $\begin{array}{l}800 \\
316 \\
160\end{array}$ & $\begin{array}{l}2 r-90 \\
2 r-90 \\
Z r-90\end{array}$ & $\begin{array}{r}415000 \\
640000 \\
1040000\end{array}$ & $\begin{array}{l}6.7 \\
5.3 \\
8.6\end{array}$ \\
\hline $\begin{array}{l}800 \\
316 \\
160\end{array}$ & $\begin{array}{l}A 1-27 \\
A 1-27 \\
A 1-27\end{array}$ & $\begin{array}{l}1080000 \\
1580000 \\
2650000\end{array}$ & $\begin{array}{l}5.3 \\
5.3 \\
7.5\end{array}$ \\
\hline
\end{tabular}

\section{Discussion}

\section{IV.1 Activation Yields}

The results from ALICE and ALICE-B were identical except where $\ell$ values greater than 21 were invoked. The main purpose of the calculations presented here were to show the effect of ALICE options on the results.

The calculated results of $180 \mathrm{MeV}$ protons on Al-27 shown in Fig. 2 are not compared with experiment here but follow the physical form expected. Each activated element has an isotopic distribution that peaks at a mass value appropriate to the energetics of the reactions. The peak mass value for each element decreases about one unit for each unit of decreasing charge.

Figure 2 shows that the cross-sections for activating low $Z$ elements from a Co-59 target increases with input proton energy as more energy is available.

In Figure 3 the yield cross-sections for activation products can be seen to vary up to approximately a factor of 2 depending on the model parameters chosen. The inclusion of deuteron emission as in Case $C$ and use of a backshifted pairing term without shell corrections as in Case E produce about the same results as the reference Case $A$. The use of the backshifted pairing terms with shell corrections as in Case $B$ and the optical model calculation of the inverse reaction cross-section as in Case $D$ lowers the activation cross-section of $\mathrm{Co-56}$ and raises the activation cross-sections of $M n-54$ and $V-50$ compared to the reference Case A. 


\section{IV.2 Neutron Emission Double Differential Cross-Sections}

Detailed differences among the calculations submitted can be observed by examining Figures 6 through 10 . Some overall conclusions, will be attempted here. In general, the calculated emitted neutron energy distribution shows a quasielastic peak, i.e., the apparent elastic scattering of the incident proton with a target neutron, in the forward angles. For small scattering angles the peak is sharp and near the incident proton energy. As the emission angle increases the peak is broadened and occurs at a lower energy as expected from the kinetics of elastic reactions.

The two sets of HETC calculations submitted can be compared at 160 , 318, and $800 \mathrm{MeV}$. The calculated shapes are similar and the corrected normalization HETC-H calculations and the HETC-F calculations are in general agreement.

At back angles PNEM calculates more high energy neutrons than calculated by the other codes.

There is good overall agreement in magnitude among the codes at forward angles. Differences, where they occur, at the forward angles, are greatest for mid energy neutrons.

The differences that occur in the double differential angular and energy distributions are primarily due to differences among the code models. PNEM, because of its empirically determined parameters, calculates a greater high energy neutron emission at back angles than the other codes. At high incident energies the back angle high energy neutron emission from HETC calculations is greater than that from ALICE-B. The HETC morieling includes more particle-particle kinetics than the ALICE modeling. The development of HETC has been primarily based on physics data above $100 \mathrm{MeV}$ whereas the development of ALICE has been primarily based on physics data helow $100 \mathrm{MeV}$. Although the HETC model calculations are expected to be valid only above 150 $\mathrm{MeV}$ where the nucleon de Broglie wave length is lower than the mean free path in the nucleus, at least for these benchmarks, there are no large differences between the ALICE and HETC-F results at 35 and $80 \mathrm{MeV}$.

The angular integrated energy distributions of Fig. 11 show closer agreement among the methods than shown in individual double differential views. The methods agree in magnitude except for $\mathrm{Al}$ at 35 and $80 \mathrm{MeV}$ where the PNEM extrapolation of high energy systematics to low energy 1 ies below the other calculations. At high energies the preequilibrium and evaporation components of the ALICE-B calculations are clearly visible. 
The integrated total $(p, x n)$ cross-sections are mostly determined by the nearly isotropic low energy neutron evaporation spectrum that peaks about $1 \mathrm{MeV}$ and are not strongly influenced by the major differences among the methods that occur at back angles where the cross-sections are small. The percentage unweighted uncertainty in Table $V I$ is less than $30 \%$ except for $\mathrm{Zr}$ at $800 \mathrm{MeV}$ and $\mathrm{Pb}$ at 35, 80, and $800 \mathrm{MeV}$. The energy at which the best overall agreement is obtained is $160 \mathrm{MeV}$ and the material for which best overall agreement is obtained is Al.

\section{IV.3 Calculation Times}

Correcting for the speed differences among the computers used, the running times among the calculational methods submitted can be approximately compared. Each of the Benchmarks 3, 4, or 5 can be calculated by PNEM in the order of seconds, ALICE or ALICE-B in the order of minutes and HETC-F or HETC-H in the order of tens or hundreds of minutes.

\section{Summary}

The study illustrated the results of using a preequilibrium plus evaporation model code to calculate the activation of materials by proton bombardment and the results of three types of model codes to calculate proton induced double differential neutron emission spectra. Circulation of the draft report was useful to obtain refinements and corrections that could be included in the benchmark comparison. For double-differential data, there was generally good agreement among the codes at forward angles. At back angles, the calculated neutron emission varied widely among the codes with results from nuclear systematics signficantly above the others. The global graphical presentation of the results for double differential data together with timing data about typical computer runs gives the reader information that can be used to determine cost effective methods for his applications.

\section{Acknowledgments}

This work was supported by the Defense Nuclear Agency and the Department of Energy, Office of Energy Research. The author very much appreciated the prompt cooperation of the participants in this study and the helpful comments by M. Blann and D. Filges and his colleagues. 


\section{References}

1. S. Pearlstein, Summary of the Meeting of the Mediuni Energy Nuclear Data Working Group, May 27-29, 1987. BNL-NCS-40070, Informal Report.

2. M. Blann, Phys. Rev. C28, 1475 (1983).

3. H. W. Bertini, Phys. Rev. 188, 1711 (1969).

4. S. Pearlstein, Nucl. Sci. and Eng. 95, 116 (1987). 


\section{APPENDIX I}

Proposed MENDWG Benchmark Problem

Activation Yields

$$
\begin{aligned}
& 180 \mathrm{MeV} p+{ }^{27} \mathrm{Al} \\
& 40 \mathrm{MeV}+200 \mathrm{MeV} p+{ }^{59} \mathrm{Co}(\mathrm{a} 11 \text { yields }) \\
& E_{p} \text { step }=10 \mathrm{MeV}, i . e ., E_{p}=40,50,60 \ldots .200
\end{aligned}
$$

\section{Double Differential Cross-section for Neutron Emission}

$$
d^{2} \sigma / d \varepsilon d \Omega(p, x n) \text { inclusive }
$$

Targets $27 \mathrm{Al},{ }^{90} \mathrm{Zr}, 208_{\mathrm{Pb}}$

$$
\begin{aligned}
E_{p}= & 35 \mathrm{MeV}-\theta \text { step }=5^{\circ}, \text { de bin }=5^{\circ}, \theta \text { center }=2.5^{\circ}, \\
& i, e ., \theta=2.5^{\circ}, 7.5^{\circ}, 12.5^{\circ}, \ldots . . ; ; \\
& E^{\prime} \text { step }=5 \mathrm{MeV}, \mathrm{dE}^{\prime} \text { bin }=1 \mathrm{MeV}, \mathrm{E}^{\prime} \text { center }=5 \mathrm{MeV}, \\
& \text { i.e., } E^{\prime}=5,10,15 \ldots . \mathrm{MeV} .
\end{aligned}
$$
$\begin{aligned} E_{p}= & 80,160 \mathrm{MeV}-\theta \text { step }=5^{\circ}, \text { bin }=5^{\circ}, \theta \text { center }=2.5^{\circ} \text {, } \\ & i . e ., \theta=2.5^{\circ}, 7.5^{\circ}, 12.5^{\circ} \ldots \ldots ;\end{aligned}$

$E^{\prime}$ step $=10 \mathrm{MeV}, \mathrm{dE}^{\prime}$ bin $=1 \mathrm{MeV}, \mathrm{E}^{\prime}$ center $=5 \mathrm{MeV}$, i.e., $E^{\prime}=5,15,25 \ldots .$. MeV.

$E_{p}=318 \mathrm{MeV}-\theta$ step $=3^{\circ}$ if possible, $\infty$ bin $=3^{\circ}, \theta$ center $=1.5^{\circ}$ i.e., $\theta=1.5^{\circ}, 4.5^{\circ}, 7.5^{\circ} \ldots \ldots$;

$\mathrm{E}^{\prime}$ step $=20 \mathrm{MeV}, \mathrm{dE}^{\prime}$ bin $=1 \mathrm{MeV}, \mathrm{E}^{\prime}$ center $=5 \mathrm{MeV}$ i.e., $E^{\prime}=5,25,45 \ldots . . M$ MeV.

$$
\begin{aligned}
E_{p}= & 800 \mathrm{MeV}-\theta \text { step }=3^{\circ} \text { if possible, do bin }=3^{\circ}, \theta \text { center }=1.5^{\circ} \\
& i . e ., \theta=1.5^{\circ}, 4.5^{\circ}, 7.5^{\circ} \ldots \ldots \text {; } \\
& E^{i} \text { step }=50 \mathrm{MeV}, \mathrm{dE}^{i}=1 \mathrm{MeV} \text {. }{ }^{\prime} \text { center }=50 \mathrm{MeV} \\
& \text { i.e., } E^{\prime}=50,100,150 \ldots \ldots \mathrm{MeV} .
\end{aligned}
$$

Results are to be sent to $S$. Pearlstein, National Nuclear Data Center, Building 1970, Upton, NY 11973, with a description or reference of the code, input parameters, and other relevant factors. The output must be in tabular form to facilitate comparison with other calculations. To be included in this comparison results should be received by 1 November 1987 . 


\section{Appendix II - Code Abstracts}

II.1 PNEM

1. Identification - PNEM - High energy double differential neutron emission and other proton and neutron induced cross-sections.

2. Computer for which software is written and other machine versions available. - DEC 10

3. Description - For high energy incident protons and neutrons, the code calculates double differential neutron emission, differential nuclear elastic, total (for neutrons) and scattering cross-sections. Angular integrated, energy integrated, and angular and integrated neutron emission cross-sections are optionally calculated.

4. Method of Solution - Parametric fits to the systematics of nuclear data.

5. Status - Preliminary version PNEM1 first available in November 1985. Second version PNEM2 available in July 1985, revised in September 1987. PNEM2 is being field tested by a few collaborators and has not been distributed for general use.

6. Restrictions - Nuclear mass range 6 to 265.

7. References -

Primary reference -

S. Pearlstein, Nucl. Sci. Eng. 95, 116-127 (1987).

Secondary references -

J. Letaw, et. al., Astrophys. J. Supp. Series 51, 271-276 (1983). Ange $1 \mathrm{i}$ and J. Csikai, Nucl. Phys. A170, 577-583 (1971).

S. Pearlstein, Nucl. Sci. Eng. 49, 162-171 (1972).

8. Hardware Requirements - Ordinary.

9. Programming Language - FORTRAN 77.

10. Operating System requirements - Ordinary.

11. Name and establishment of author or submitter -

Sol Pearlstein

National Nuclear Data Center

Brookhaven National Laboratory

Upton, New York 11973

(516) $282-2901$, FTS 666-2901

12. Material Available - See Status. 


\begin{abstract}
II.2 ALICE
1. Identification - ALICE/LIVERMORE $85 / 300$ precompound compound decay.

2. Computer for which Software is Written and Other Machine Versions Available - CDC 6700 (designed to be machine-dependent).

3. Description - ALICE/LIVERMORE 85 calculates particle spectra and yields for precompound plus compound decay, including fission competition. Multiple decay of neutron $(n)$, proton $(p)$, alpha, and deuteron (d) particles may be considered. The program can perform a standard Weisskopf-Ewing evaporation calculation with multiple particle emission or an s-wave approximation calculation which gives an upper limit to the enhancement of gamma ray de-excitation due to angular momentum effects. The evaporation calculation can include fission competition according to the Bohr-wheeler approach, using angular momentum dependent ground state and saddle point energies. (The latter values are derived from Cohen, et al, rotating liquid drop model or Sierks finite range model.) Provision to permit precompound emission via the hybrid and geometry-dependent hybrid models is included.
\end{abstract}

4. Method of Solution - In the Weisskopf evaporation calculation, the inverse reaction cross sections may be provided as input data or computed with a classical sharp cutoff algorithm or an optical model routine. In the s-wave approximation the calculation is performed for every partial wave in the entrance channel. The transmission coefficients for the entrance channel partial waves may be provided as input data or by the parabolic model or optical model calculation. The Myers-Swiatecki/Lysekil mass formula is used to calculate the $q$ value for formation of the compound nucleus and the $n$, $p$, alpha, and $d$ binding energies for all nuclides of interest in the evaporation chain, or experimental masses (available in a library subroutine) can be used.

5. Restrictions on the Complexity of the Problem - Excitation energies of the compound nucleus up to $200 \mathrm{MeV}$ can be considred in the Weisskopf evaporation calculation. In the s-wave approximation it is assumed that the rotational energy for each partial wave is irrevocably committed to rotational motion and, therefore, unavailable for particle emission.

6. Timing - The sample problem execution time is approximately 10 seconds on a $\operatorname{CDC} 7600$ and 40 seconds on an IBM 3033.

7. Unusual Features of the Software - None, standard FORTRAN IV.

8. Related and Auxiliary Software - ALICE/LIVERMORE 85 is a revision of the earlier ALICE and OVERLAID ALICE codes. 
9. Status - Abstract first distributed April 1984.

CDC 7600 version submitted November 1983, sample problem executed by NESC December 1983 on an IBM 3033.

10. References - M. Blann and J. Bisplinghoff, CODE ALICE/LIVERMORE 82, UCID-19614 November 5, 1982; M. Blann, CODE ALICE/85/300, UCID 20169 (October 1984).

ALICE/LIVERMORE 82, NESC No. 1036, ALICE/LIVERMORE 82 Tape Description and Implementation Information, National Energy Software Center Note 84-28, April 30, 1984.

M. Blann, OVERLAID ALICE: A Statistical Model Computer Code including Fission and Preequilibrium Models, C00-3494-29, August 1976.

11. Hardware Requirements - NESC used approximately $244 \mathrm{~K}$ bytes on an IBM 3033 to execute the sample problem.

12. Programming Language - FORTRAN IV.

13. Operating System - SCOPE (CDC 7600), OS/MVT (IBM 3033).

14. Other Programming or Operating Information or Restrictions - The FORMAT statements contain " delimiters.

15. Name and Establishment of Author or Contrivutor -

M. Blann

Lawrence Livermore National Laboratory

J. Bisplinghoff

Institut fuer Strahlen und Kernphysik

Bonn University

Germany

16. Material Available

Source

Sample problem input

Sample problem output

Reference report, UCID 20169

17. Keywords - evaporation model, compound nucleus reactions, precompound-nucleus emission, Weisskopf model, Bohr-Wheeler theory, nuclear models.

18. Sponsor - DOE Office of High Energy and Nuclear Physics. 
I I.3 ALICE-B

1. Identification - ALICE-B

2. Computer for which Software is Written - DEC-10, VAX-780

3. Description - Derived from ALICE/LIVERMORE 85. Its algorithms extended to $1000 \mathrm{MeV}$. The user should be warned that the physics may not be applicable at these energies. Partial wave analysis extended to $\ell=70$. Number of energy bins for angular distributions increased. Above $300 \mathrm{MeV}$ the optical model cross-section is corrected.

4. Unusual Features of the Software - G-Floating point arithmetic.

5. Related and Auxiliary Software - ALICE-B is an informal revision of ALICE/LIVERMORE 85.

6. Status - Experimental. All changes communicated to M. Blann, author of ALICE/LIVERMORE $85 / 300$ for possible inclusion in future versions.

7. References - S. Pearlstein, private communication.

8. Programing Language - FORTRAN IV.

9. Name and Establishment of Author or Contributor -

S. Pearlstein

Building 1970

Brookhayen National Laboratory Upton, NY 11973

10. Material Available - None.

11. Keywords - evaporation model, compound-nucleus reactions, precompound-nucleus emissior, Weisskopf model, Bohr-Wheeler theory, nuclear models. 


\section{II.4 HETC-CCC -178}

Since HETC-F and HETC-H make extensive use of RSIC Code Package -178 , information on RSIC Code Package CCC-178 is included.

1. Identification - HETC: Monte Carlo High Energy Nucleon Meson Transport Code System.

$\begin{array}{ll}\text { Auxiliary } & \text { Routines - } \\ \text { COPY: } & \text { Utility routine } \\ \text { PUNCROSS: } & \text { Cross Section Handling Code } \\ \text { HET: } & \text { Nucleon-Meson Transport Code } \\ \text { NMTA: } & \text { Data Processing Code } \\ \text { EHET: } & \text { Edit Code } \\ \text { XSECT: } & \text { Cross Section Data Generator } \\ \text { OSR: } & \text { Modified Monte Carlo Code (CCC-17). } \\ \text { O5RA: } & \text { O5R Sample Problem Analysis Code } \\ \text { HETC is basically an extension of CCC-161/NMTC. }\end{array}$

2. Contributor - Oak Ridge National Laboratory, Oak Ridge, Tennessee.

3. Coding Language and Computer - FORTRAN IV; IBM 360/370.

4. Nature of Problem Solved - HETC ir a Monte Carlo transport code for computing the properties of high-energy nucleon-meson cascades in matter. It is basically an extension of CCC-161/NMTC (va?id for energies \& $3 \mathrm{GeV}$ ) to allow particle transport up to several hundred GeV.

The source particle description is specified in a user-written subroutine and may be arbitrarily distributed in energy, direction, and space. Proton, neutron, $\mathrm{pi}^{+}, \mathrm{pi}^{-}, \mathrm{mu}^{+}$, and $\mathrm{mu}^{-}$sources are allowed.

Although the maximum allowable source particle energies are not well defined, HETC has been run successfully for energies up to $1 \mathrm{TeV}$. However, because of the lack of experimental data at very high energies, comparison of HETC results with experimental data have been made only for energies $<30 \mathrm{GeV}$.

The code stores on magnetic tape a complete description of each "event" (nuclear interaction, geometry boundary crossing, pion decay, etc.) that occurs during the transport process. This information is then read and processed by user-written analysis programs to obtain results for a particular problem.

Differential cross sections for nucleon-nucleus and pion-nucleus nonelastic collisions are not required as input since they are, in effect, computed in the course of the transport calculation using the intranuclear-cascade-extrapolation-evaporation model. 
Since HET provides a complete description of the low energy $\ll 15$ $\mathrm{MeV}$ ) neutron production, HET may be readily coupled with codes other than 05R (e.g., CCC-254/ANISN, CCC-209/DOT, CCC-203/MORSE) to obtain the low energy neutron transport.

5. Method of Solution - HETC consists of two basic transport codes: HET and a modified version of 05R. HET transport particles in the energy range from the source-particle energy down to a specified energy cutoff, which is commonly taken to be $15 \mathrm{MeV}$ for protons and neutrons, $2 \mathrm{MeV}$ for charged pions, and $0.2 \mathrm{MeV}$ for muons. Neutrons produced in HET below the cutoff energy are transported via the 05R code.

The description of nonelastic-collision pioducts in HET is obtained using an intranuclear-cascade-extrapolation-evaporation model. At each nonelastic collision a calculation is performed using subprogram versions of Bertini and Guthrie's latest intranuclear-cascade program (Nucl. Phys. A169, 670 (1971)) and Guthrie's evaporation program (ORNL-TM-3119) to determine the energy and direction of emitted cascade nucleons and pions and evaporated nucleons, deuterons, tritons, (ORNL-TM-3667) He-3's, and alpha particles and the mass, charge the recoil energy of the residual nucleus. For nonelastic collisions $<3 \mathrm{GeV}$, these intranuclear-cascade-evaporation results are used directly; for nonelastic collisions $>\mathrm{GeV}$, the results from an intranuclear-cascade-evaporation calculation at approximately $3 \mathrm{GeV}$ are scaled to the proper energy using the extrapolation method of Gabriel, Alsmiller, Jr., and Guthrie. Nonelastic collisions with hydrogen nuclei are treated using experimental data and the calculation method of Gabriel, Santoro, and Barish (ORNL-TM-3615). Nonelastic collisions in $05 R$ are treated using the evaporation model in conjunction with experimental cross sections. Experimental data are used for elastic-collision cross sections.

Charged-pion and muon decay in flight and at rest are taken into account using known lifetimes.

6. Restrictions or Limitations - Present dimensions restrict the number of different media to 15 or less and the number of types of nuclei per medium to 11 or less. Virtually arbitrary geometries may be specified.

7. Typical Running Time - Running time is extremely problem dependent. A sample problem included with the code documentation required approximately 10 minutes on the IBM $360 / 91$ and approximately 3 times longer on the IBM $360 / 75$.

8. Computer Hardware Requirements - HETC is operabl: on the IBM $360 / 370$ computers. Approximately $1000 \mathrm{~K}$ bytes of memory and 4 peripheral storage devices are required. 
9. Computer Software Requirements - A FORTRAN H compiler is required.

10. References

K. C. Chandler and T. W. Armstrong, "Operating Insíructions for the High-Energy Nucleon-Meson Transport Code HETC," ORNL-4744 (January 1972).

W. A. Coleman and T. W. Armstrong, "The Nucleon-Meson Transport Code NMTC," ORNL-4606 (October 1970).

T. W. Armstrong and K. C. Chandler, "NMTA--Analysis Subroutines for the Nucleon-Meson Transport Code NMTC," ORNL-4736 (November 1971).

D. C. Irving, R. M. Freestone, Jr., and F. B. K. Kam, "05R, A General-Purpose Monte Carlo Transport Code," ORNL-3622 (February 1965).

D. C. Irving, W. E. Kinney, and Pat Rea, "LEGENDRE, A Program to Calculate Legendre Coefficients from Angular Distributions," ORNL-TM1241 (November 1965).

D. C. Irving, V. R. Cain, and R. M. Freestone, "An Amplification of Selected Portions of the O5R Monte Carlo Code User's Manual," ORNLTM-2601 (May 1969).

T. W. Armstrong, R. G. Alsmiller, Jr., K. C. Chandler, and B. L. Bishop, "Monte Carlo Calculation of High-Energy Nucleon-Meson Cascades and Comparison with Experiment," ORNL-TM-3667 (January 1972).

James B. Langworthy, "An Organizational Description of High Energy Transport Code (HETC)," NRL Memorandum Report 2729 (June 1974).

11. Contents of Code Package - Included are the referenced documents and a reel of magnetic tape which contains source decks, random number generators, input data written in BCD/EBCDIC card images, plus output written in list format; total records $62,533(A)$ and 5486 (B).

12. Date of Abstract - December 1974; updated May 1975.

13. Keywords - Monte Carlo, nucleon; neutron; proton; meson; complex geometry; high energy.

14. Availability - Contact Radiation Shielding Information Center, Oak Ridge National Laboratory, P.0. Box X, Oak Ridge, TN 37831-6362, Telephone (615) 574-6176. 
II.5 HETC-H Description and Calculational Details

KFA-Version of HETC and Description of the Procedure to Calculate Cross-Sections

HETC-RFA-2 in the HERMES system

To simulate the histories, i.e. production, interaction with matter and transport, of high energetic particles the latest state-of-the-art radiation transport codes and event generators are used in HERMES. All used codes employ Monte Carlo techniques with 3 dimensional geometry description, therefore, it is possible to treat in detail the reaction mechanism and the transport of the high energy particles as well as of the lowenergy particles created. An overview of the main physics models is given in Ref. 1. Some details about the hadron cascade module HETC-KFA-2 used at KFA are given below.

HETC (High Energy Transport Code) is implemented in a version based on the original coding given in Ref. 2. Other important references about the models are given in Refs. 3 and 4.

Name: HETC-KFA-2

(High-Energy-Radiation-Transport-Code, KFA Version 2)

Implementation: based on Ref. 2 (Report ORNL-4744) Extension: FORTRAN77 compatible on IBM 3081 and CRAY-XMP2 2

Particles

n, p, $\pi^{ \pm}, \mu^{ \pm}$

transported:

Extension:

d, $t, \mathrm{He}^{3}, \alpha$, heavy ions $\mathrm{A}<20$

Mechanism:

- collision model - Intranuclear-CascadeEvaporation (INCE) (for $p, n, \pi^{ \pm} ; \pi^{-}$capture at rest; nonelastic nuclear collisions, nonelastic hydrogen collisions; $\pi^{0}, d, t, \mathrm{He}^{3}, a-$ production: residual nuclei and residual excitation energy)

- charged particle ionization energy loss $(\mathrm{dE} / \mathrm{dx})$

- primary particle small angle multiple

- Coulomb scattering

- decay (weighted fashion) for $\pi \pm$ and $\mu^{ \pm}$in flight and at rest 
Extensions :

Geometry:

Extension :

Additional

Extensions :
- scaling model - automatic parameter update for nonelastic collisions above $3.5 \mathrm{GeV}$

(limited energy range for scaling

pn $-E_{\max } \leqslant 25 \mathrm{GeV}$

$\pi^{ \pm}-E_{\max } \leqslant 15 \mathrm{GeV}$ )

- elastic collision module for $p / n$ elastic nucleus collisions upto $20 \mathrm{GeV}$

- high energy fission included in evaporation model

- automatic parameter update for $B_{0}$ in level density formula

- nonisotropic angular distribution of evaporated particle

- analog decay for $\pi^{ \pm}$

standard CG geometry interface

CG-KFA geometry module

submission of particles and nuclei for further processing structure of HERMES structure of HERMES submission files

The cross-section calculations were performed w1th the HETC-KEA-2 for the present benchmark with a socalled thin target setup. This means that only the intranuclear cascade evaporation model is used. The emitted neutrons in this model are not able to undergo further nuclear reactions with other nuclei. For all targets the variable Bo-option of the code and a isotropic emission for evaporation was assumed while high energy fission was excluded. All cases were run with about 5 to 7 hours computer $t$ ime on IBM-3081. The number of incident protons (Table 3 ) and also the binning of the energy intervals were chosen in a way that even at energies and angles with a low probability for neutron emission an acceptable neutron yield could be determined. This means, that in the average at every energy-angle-bin at least more than 100 emitted neutrons were sampled.

In the current version of HETC-KFA-2 neutron production spectra are determined by defining yield detectors for the analysis of the specific nuclear reaction. A detector has to be understood as an analysis about some criteria for example the type, energy and emission angle of particles (Table 1). Therefore, the HETC output contains the neutron yield in predefined energy and angle ranges normalized to the number of source protons and referred to a nuclear density of unity. The double differential cross-section is then calculated by the following procedure. 


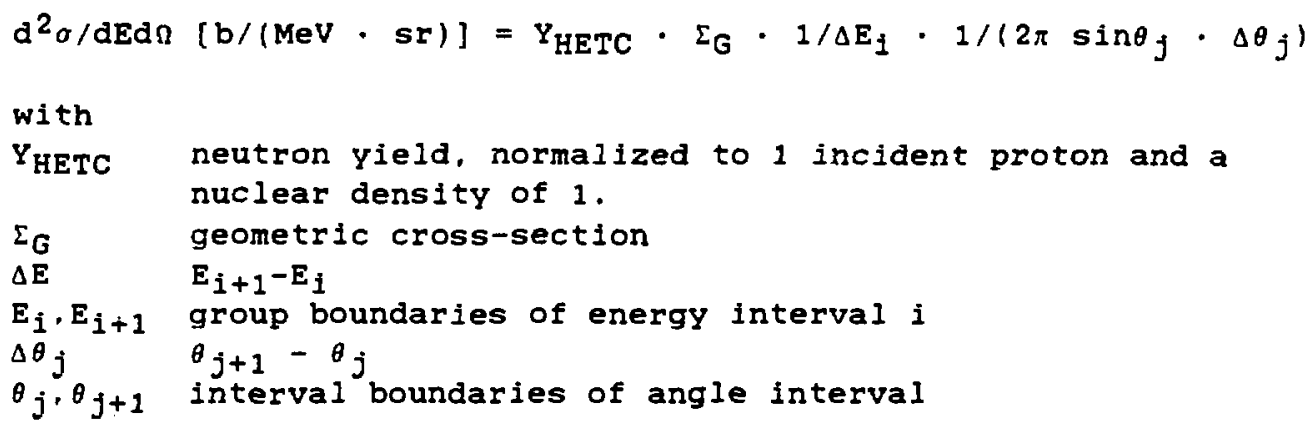

The transparency factor in the HETC-KFA-2 has not to be considered any more like in former versions. The values of geometric cross-sections are given in Table 2 .

\begin{tabular}{ccccc}
\hline $\begin{array}{c}\text { Energy } \\
{[\mathrm{MeV}]} \\
{[\mathrm{MeV}]}\end{array}$ & $\begin{array}{c}\text { No. of } \\
\text { Energy-bins }\end{array}$ & $\begin{array}{c}\text { Width of } \\
\text { Energy-bins } \\
{[\mathrm{MeV}]}\end{array}$ & $\begin{array}{c}\text { No. of } \\
\text { Angle-bins }\end{array}$ & $\begin{array}{c}\text { Width of } \\
\text { angle-bins } \\
\text { [degree }]\end{array}$ \\
\hline 160. & 16. & 10. & 36. & 5.0 \\
318. & 31. & 10. & 60. & 3.0 \\
800. & 40. & 20. & 60. & 3.0
\end{tabular}

Table 1: Energy- and angle binning used for MENDWG benchmark problem

\begin{tabular}{lccc}
$\begin{array}{l}\text { Target } \\
\text { Nucleus }\end{array}$ & $\begin{array}{c}\text { Proton- } \\
\text { Energy } \\
{[\mathrm{MeV}]}\end{array}$ & $\begin{array}{c}\text { Geometric } \\
\text { Cross Section } \\
\text { [barn] }\end{array}$ & $\begin{array}{c}\text { Transparency } \\
\text { of the Nucleus }\end{array}$ \\
\hline $\mathrm{Al-27}$ & 160 & 1.0282 & 0.400 \\
& 318 & 1.0282 & 0.386 \\
& 800 & 1.0282 & 0.476 \\
$\mathrm{Zr}-90$ & 160 & 1.6269 & 0.560 \\
& 318 & 1.6769 & 0.548 \\
& 800 & 1.6769 & 0.623 \\
$\mathrm{~Pb}-208$ & 160 & 2.4615 & 0.662 \\
& 318 & 2.4615 & 0.647 \\
& 800 & 2.4615 & 0.706
\end{tabular}

Table 2: Geometric cross sections and transperencies of the nuclei $\mathrm{Al}-2 \mathrm{Z}, \mathrm{Zr}-90$ and $\mathrm{Pb}-208$ at the thin-target calculations with HETC/KFA-2 


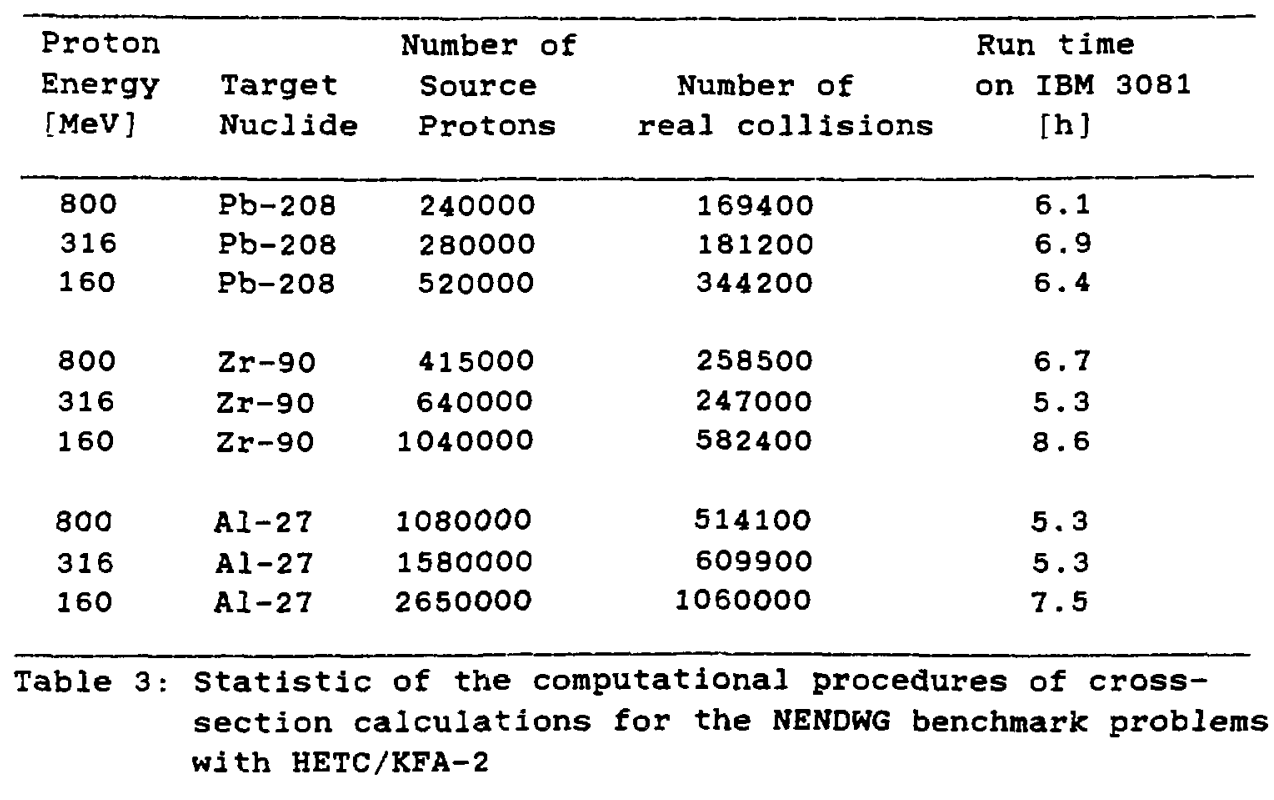


$11 /$ P. Cloth, D. Filges, R.D. Neef, G. Sterzenbach, Ch. Reul. T.W. Armstrong. B.L. Colborn, B. Anders, H. Brückmann HERMES - High Energy Radiation Monte Carlo Elaborate System

KFA Jiil-Report, in preparation

$12 /$ T.W. Armstrong. K.C. Chandler HETC, Monte-Carlo High Energy Nucleon Meson Transport Code ORNL-4744, January 1972

/3/ T.W. Armstrong, K.C. Chandler HETC - A High Energy Transport Code Nucl.Sci. and Eng. 49, 110 (1972)

14/ Monte Carlo High-Energy Nucleon-Meson Transport Code Oak Ridge National Laboratory RSIC Computer Code Collection, CCC-178 


\section{II.6 HETC-F (TIERCE)}

\section{MAYE AND TITLE}

TIERCE : (Transport des Ions et des $\underline{R}$ idiations dans les $\underline{\text { Cibles }}$ Epaisses)

Auxillary Routines :

HETC : Nucleon-Meson Transport Code (CCC-178/HETC)

EHET : Edit Code

MORSE : Modified Monte Carlo Code (CCC-203/Morse C.G)

Tierce is an extensive modification of CCC-178/HETC.

\section{CODMG LANGUAGE AND COMPUTER}

FORTRAN 77 ; IBM $3090 / 200$

\section{NATURE OF PROBLEH SOLVED}

Tierce is a Monte-Carlo transport code system for computing the properties of high-energy nucleon meson cascades in matter. It is basically an extension of CCC-178/HETC (valid for energies up to several hundred $\mathrm{GeV}$ ).

The source particle description is spécified in a user-written subroutine and may be arbitrarily distributed in energy, direction, and space. Proton neutron, $\mathrm{Pi}^{+}, \mathrm{Pi}^{-}, \mathrm{mu}^{+}$, $\mathrm{mu}^{-}$, and light ion (A $\leq 8$ ) sources are allowed.

For the target description the combinatorial geometry is used.

Each event (nuclear interaction, geometry boundary crossing, pion decay, etc...) that occurs during the transport process is analysed "on-line", according to the user choice. However, an history tape can be generated if necessary, to be read and processed "off-line" to obtain results for a particular problem. 
Differential cross sections for nucleon-nucleus and pionnucleus, nonellastic collisions are not required as input since they are in effect, computed in the course of the transport calculation using the intranuclear-cascade-evaporation model.

The low energy neutron ( $15 \mathrm{MeV}$ ) transport is processed by MORSE which is coupled with HETC.

The results obtained by the different modules can be merged and synthetized under graphic representation according to user choice.

\section{IV - METHOD OF SOLUTIOY}

TIERCE consists of two basic transport codes : HETC and a modified version of MORSE. HETC transport particles in the energy range from the source-particle energy down to a specified energy cutoff, which is commonly taken to be $15 \mathrm{MeV}$ for protons and neutrons, $2 \mathrm{MeV}$ for charged pions, and $1.6 \mathrm{MeV}$ for muons. Neutrons produced in HETC below the cutoff energy are transported via the MORSE code.

The description of nonelastic-collision products in HETC is obtained using an intranuclear-cascade-extrapolation-evaporation (with fission). For A 21 incident particle, the nucleons giving a non-elastic collision are treated under either evaporation or intranuclear-cascadeevaporation model. In the later case a calculation is performed using subprogram versions of Bertini and Guthrie's latest intranuclear-cascade program (Nucl. Phys. Al69, 670 (1971)) and Guthrie's evaporation program (ORNL-TM-3119) to determine the energy and direction of emitted cascade nucleons and pions and evaporated nucleons, deuterons, tritons, (ORNL-TM-3667) $\mathrm{He}^{3^{\prime}} \mathrm{s}$, and alpha particles and the mass, charge, recoil energy of the residual nucleus. For nonelastic collisions $\leq 3 \mathrm{GeV}$, these intranuclear-cascade-evaporation results are used directly; for nonelastic collisions $23 \mathrm{GeV}$, the results from an intranuclear-cascade-evaporation calculation at approximately $3 \mathrm{GeV}$ are scaled to the proper energy using the extrapolation method of Gabriel, Alsmiller, Jr., and Guthrie. Nonelastic collisions with hydrogen nuclei are treated using experimental data and the calculation method of Gabriel, Santoro, and Barish (ORNL-TM-3615). Nonelastic collisions in MORSE are treated using experimental neutron cross sections. Experimental data are used for elastic-collision cross sections.

Charge-pion and muon decay in flight and at rest are taken into account using known lifetimes. 


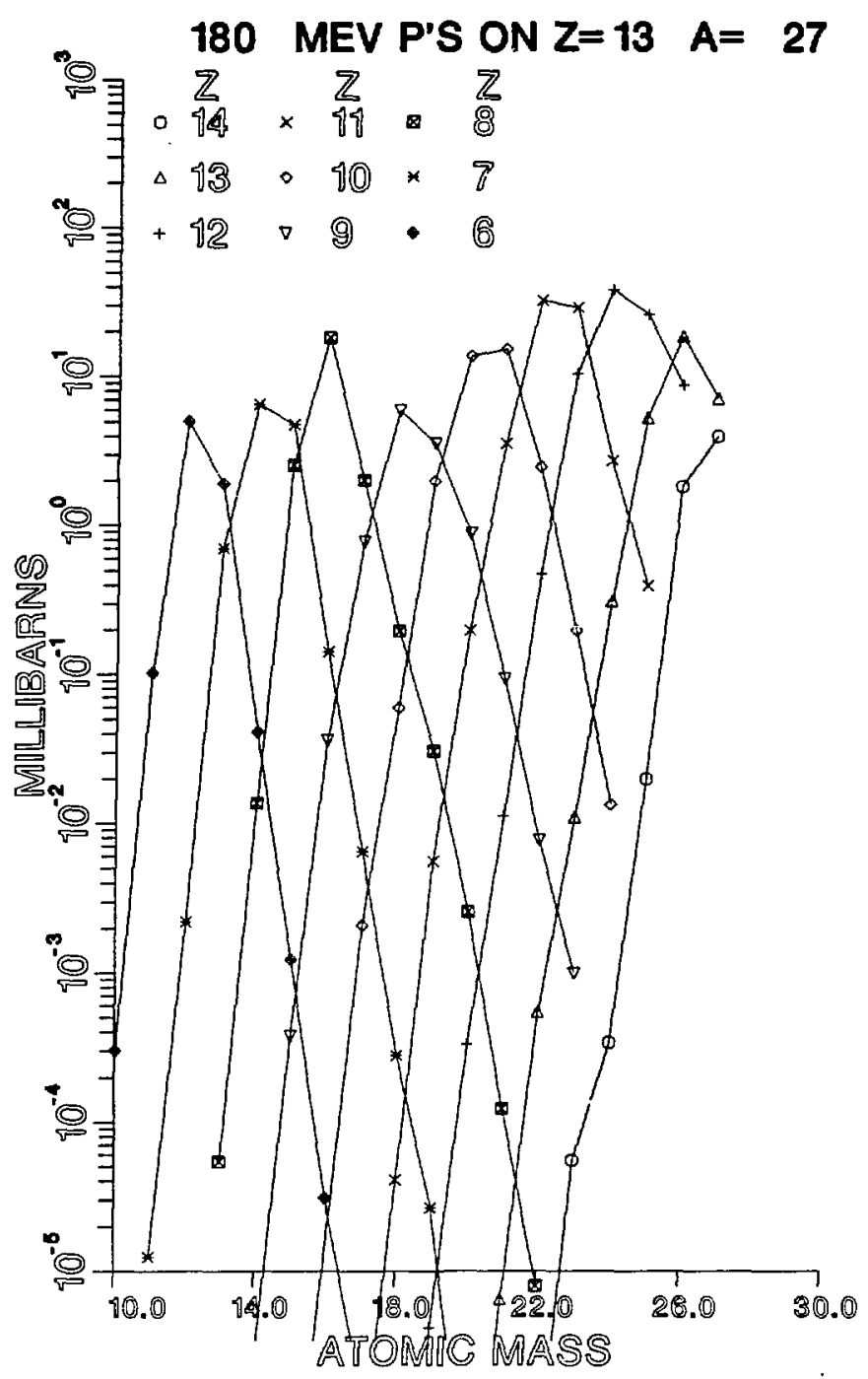

Fig. 1. Yield Cross-Sections of Activation Products from 180 MeV Protons on A]-27 
(a)

Fig. 2. Yield Cross-Sections of Activation Products from a) 120 , b) 160 , and c) $200 \mathrm{MeV}$ Protons on Co-59.

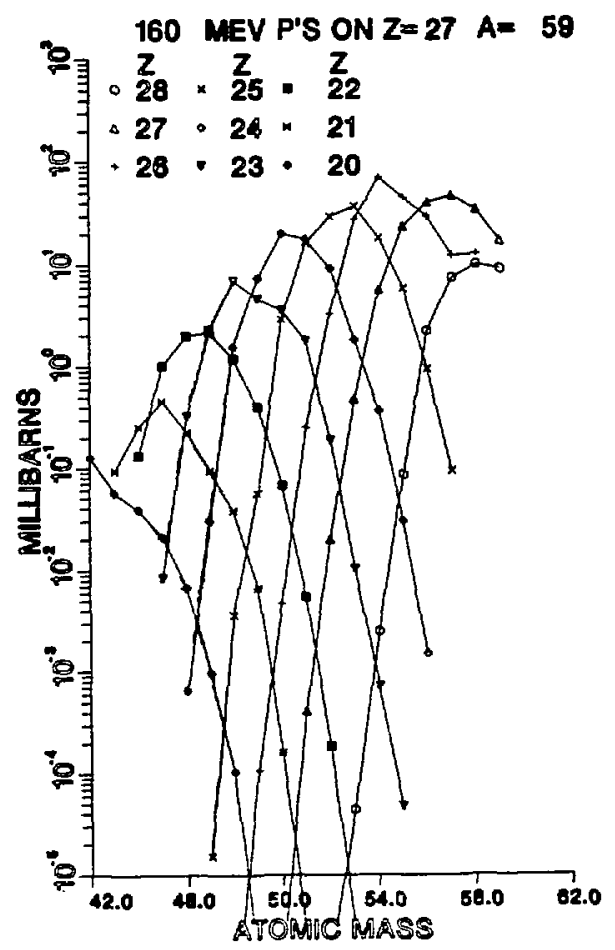

(b)
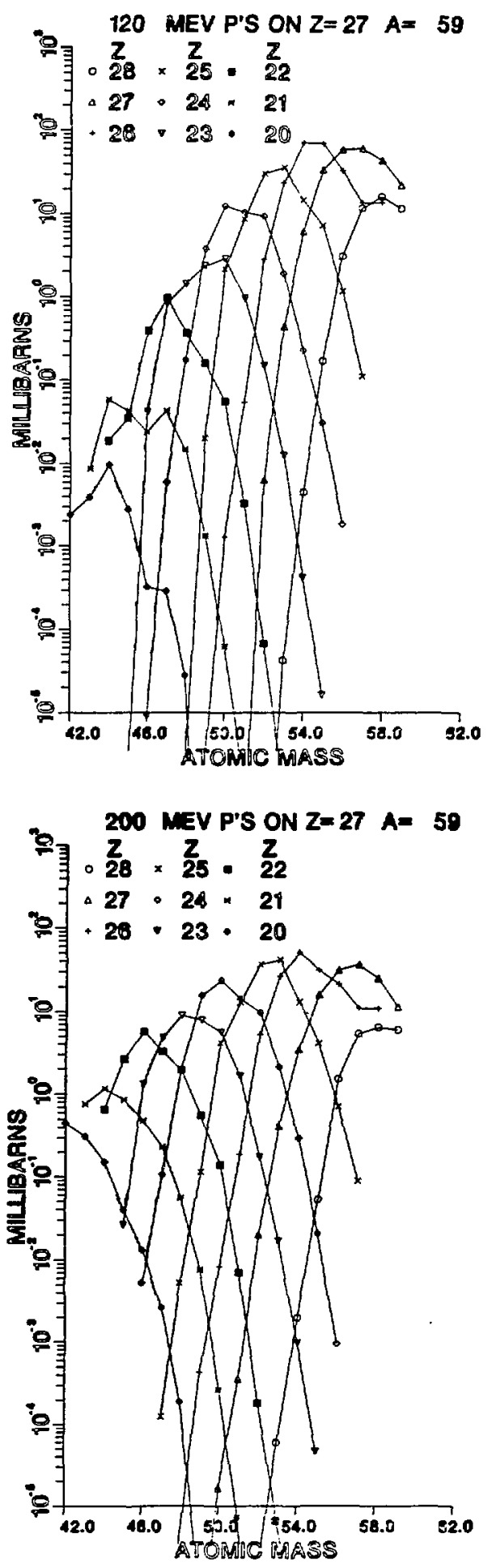

(c) 
Fig. 3. Yield Cross-Sections of Activation Products frol Protons on C0-59. See text for explanation of symbols $A$ through $E$.

a) $\mathrm{Co-56}$, b) $\mathrm{Nn}-54$, c) $\mathrm{V}-50$
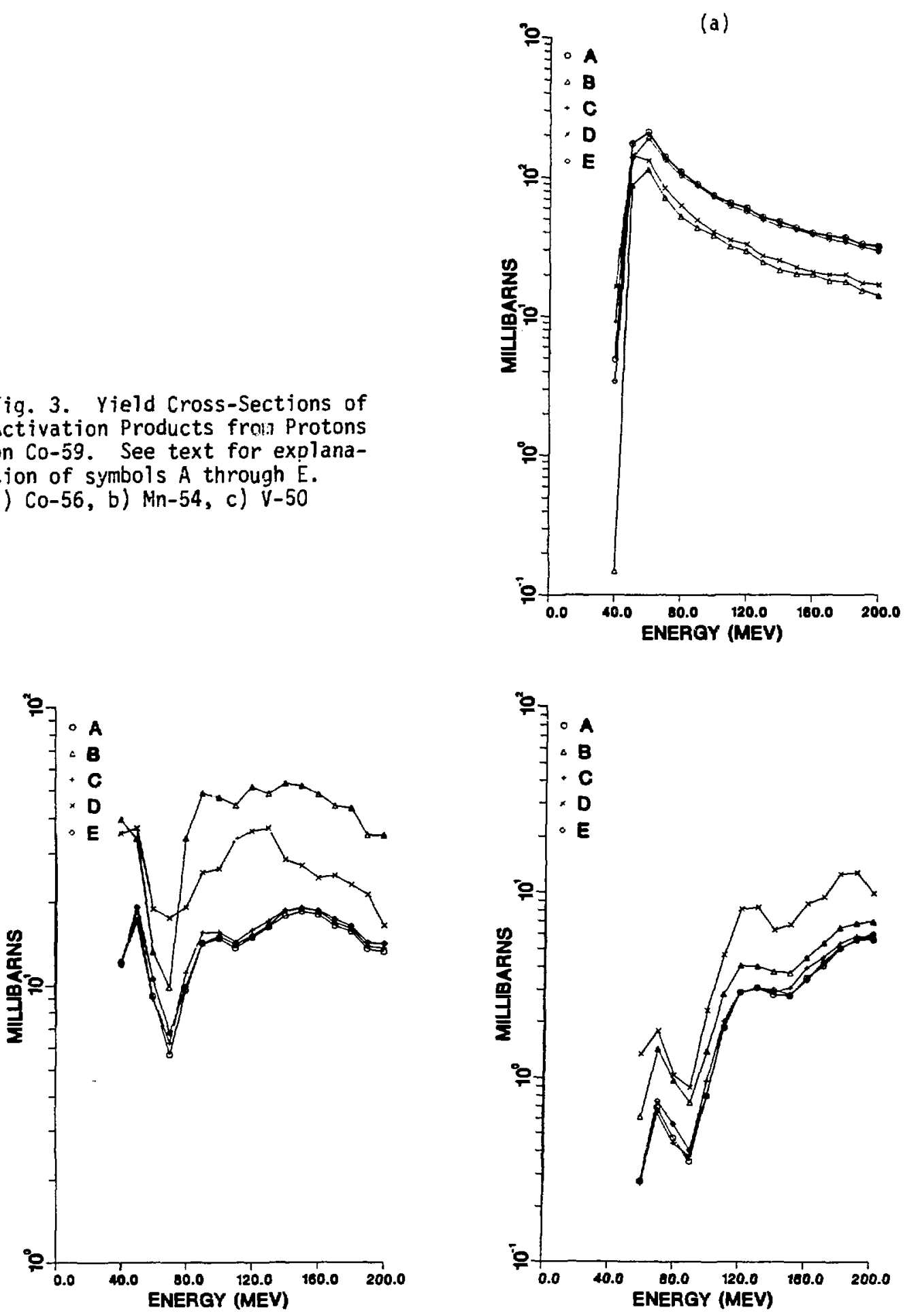

(b)

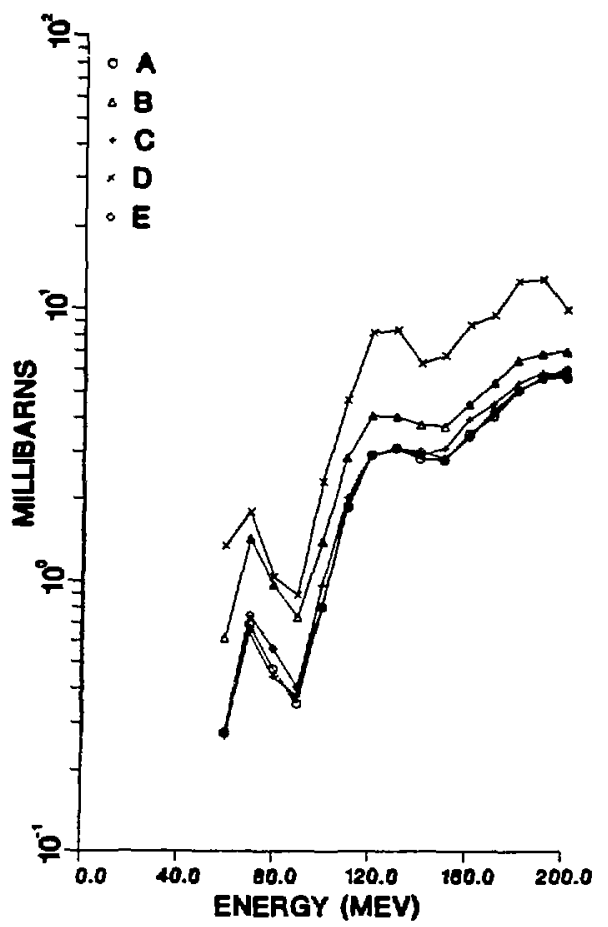

(c) 
4 (a)

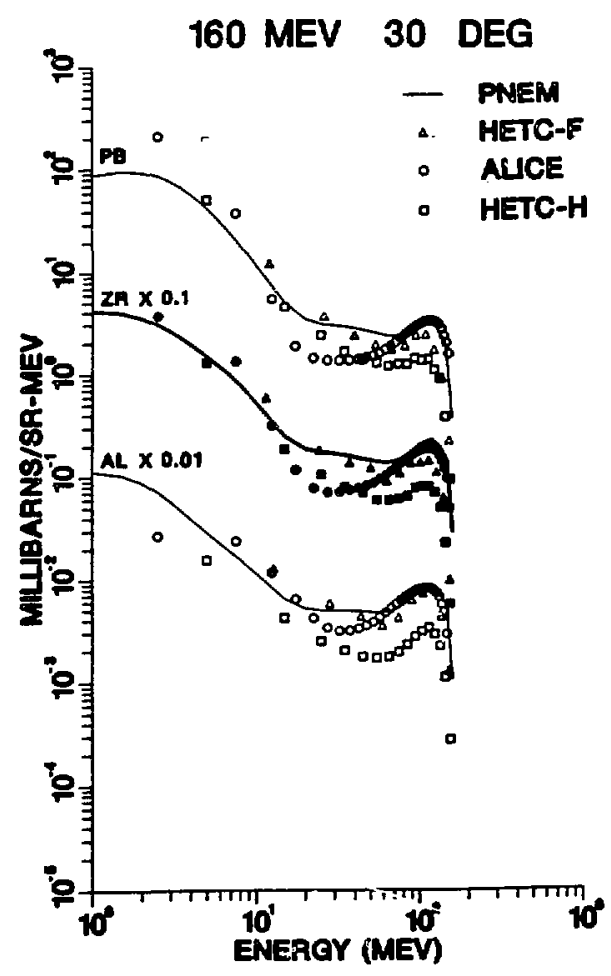

Fig. 4. Neutron energy spectra from $160 \mathrm{MeV}$ protons on $\mathrm{Al}-27, \mathrm{Zr}-90$ and $\mathrm{Pb}-208$ at an emission angle of 30 degrees 4 (a) Draft report, 4(b) Final report.

Fig. 5. Neutron energy spectra from $\mathrm{Pb}-208$. Same data as Figure 4(b) but plotted on a log-linear scale with the cross-section per unit lethargy. $4(b)$

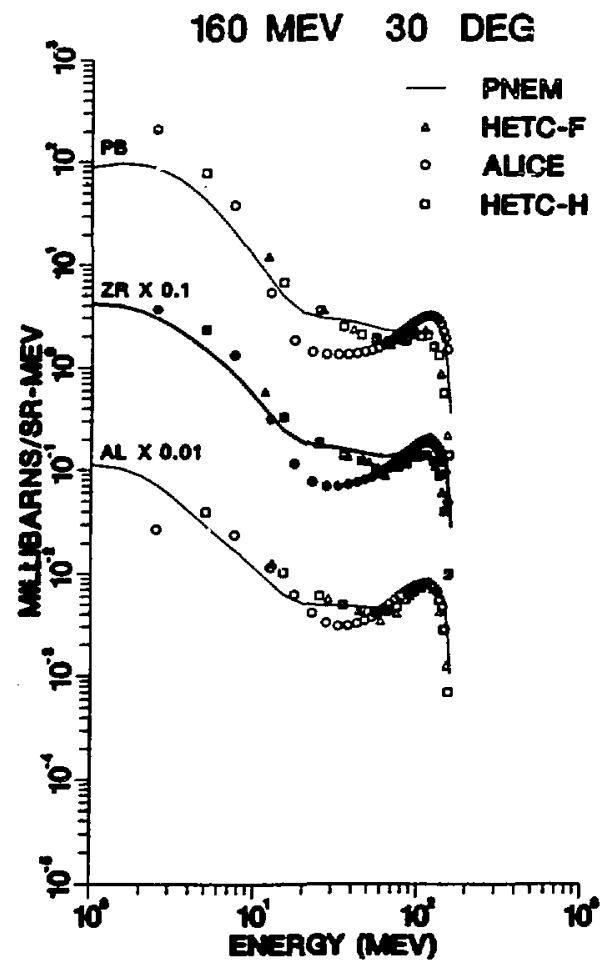

160 MEV 30 DEG

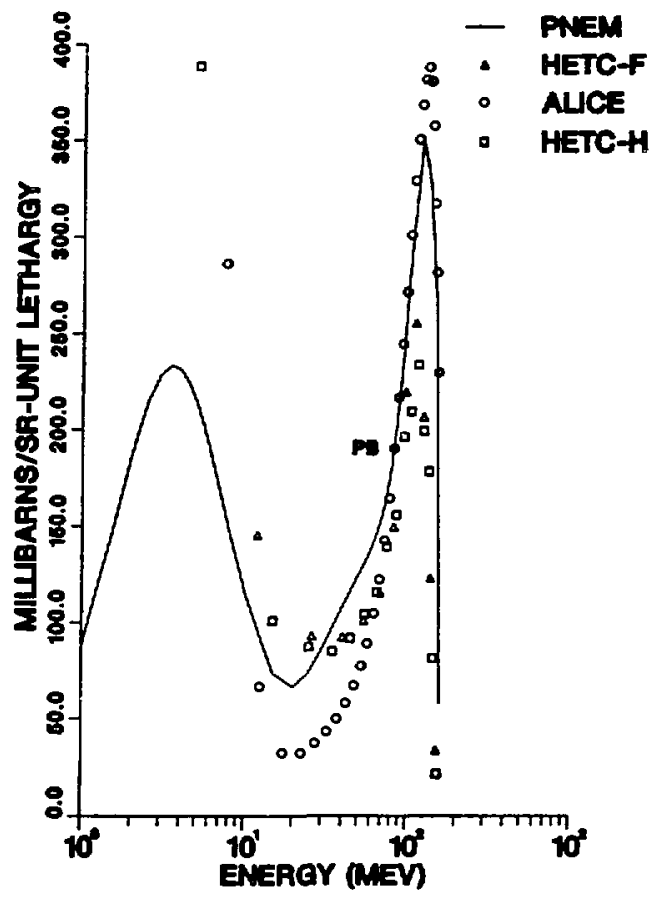


Fig. 6. Neutron energy spectra from $35 \mathrm{MeV}$ protons on A1-27, $\mathrm{Zr}-90$ and $\mathrm{Pb}-208$ at emission angles $15,30,45,60,90,120$, and 150 degrees.

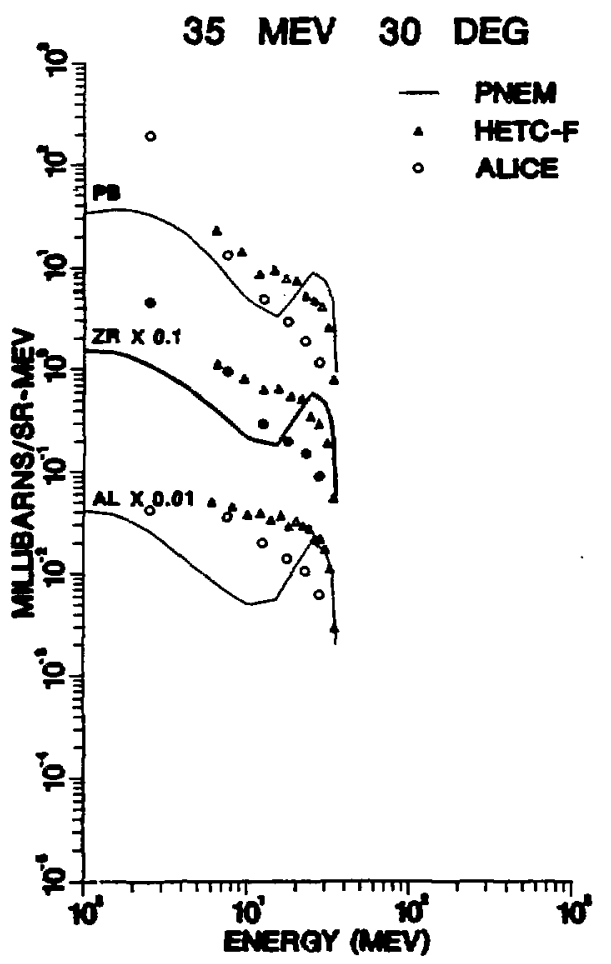

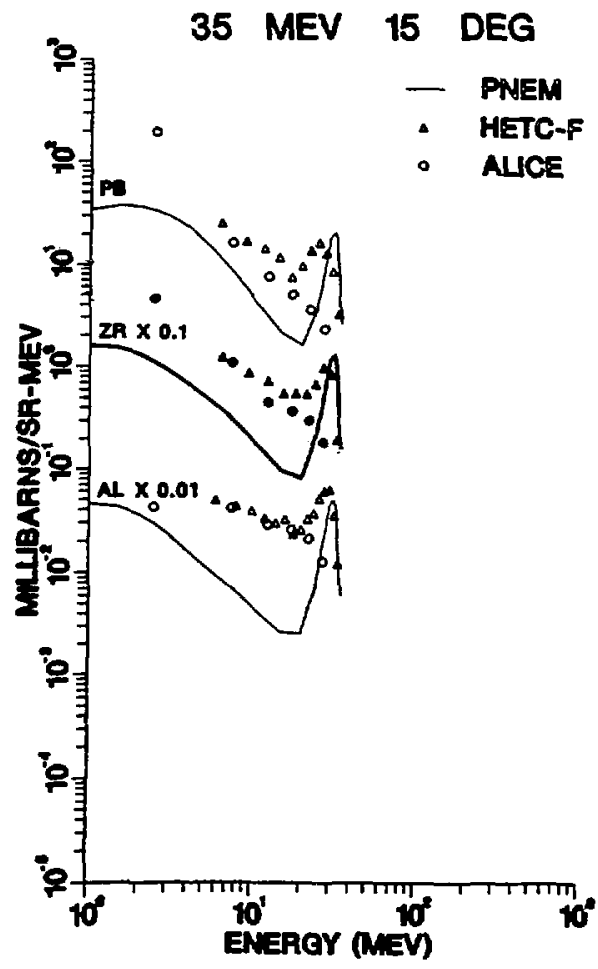

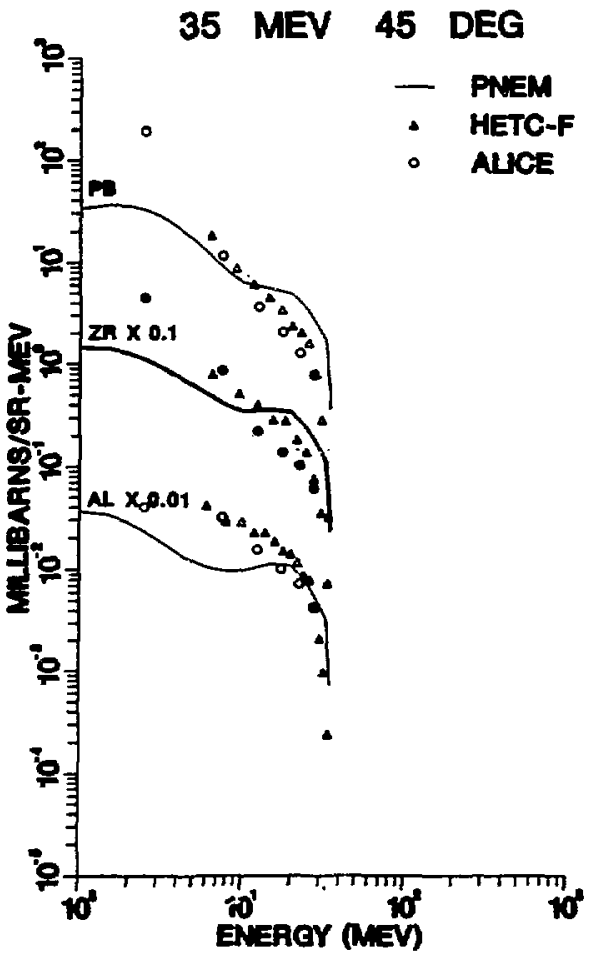


Fig. 6. cont.
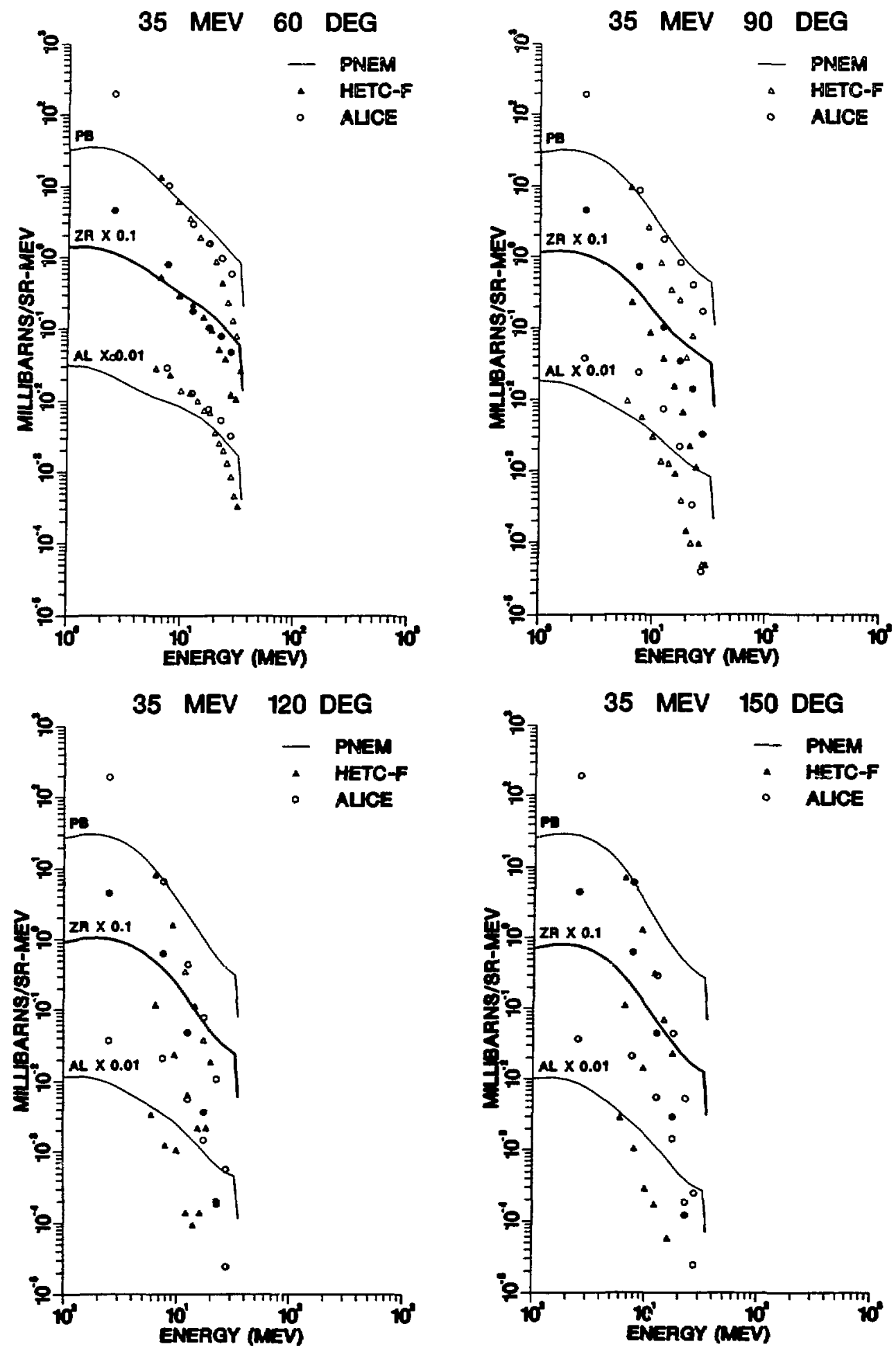
Fig. 7. Neutron energy spectra from $80 \mathrm{MeV}$ protons on $\mathrm{A}]-27$, $\mathrm{Zr}-90$ and $\mathrm{Pb}-208$ at emission angles $15,30,45,60,90,120$ and 150 degrees.

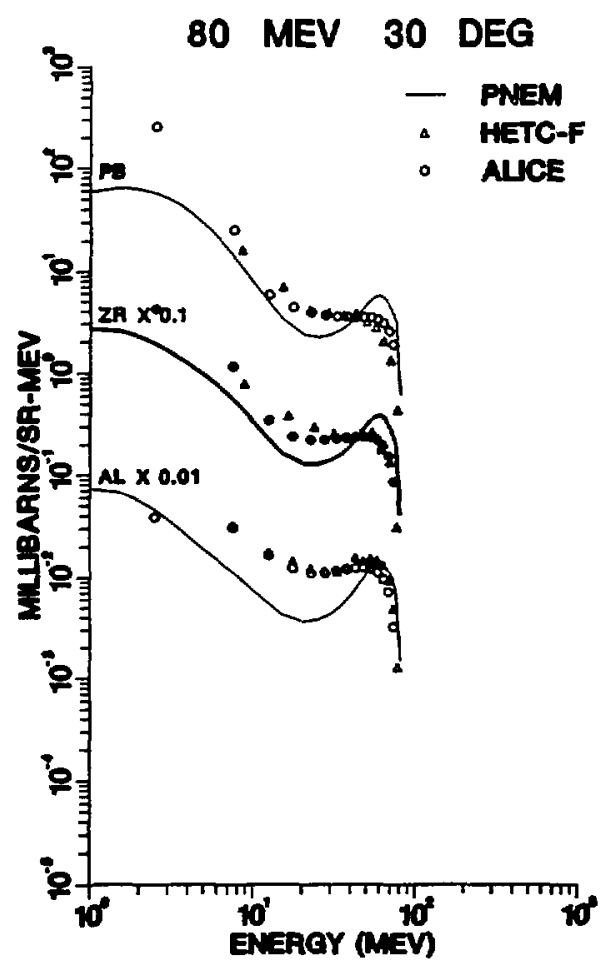

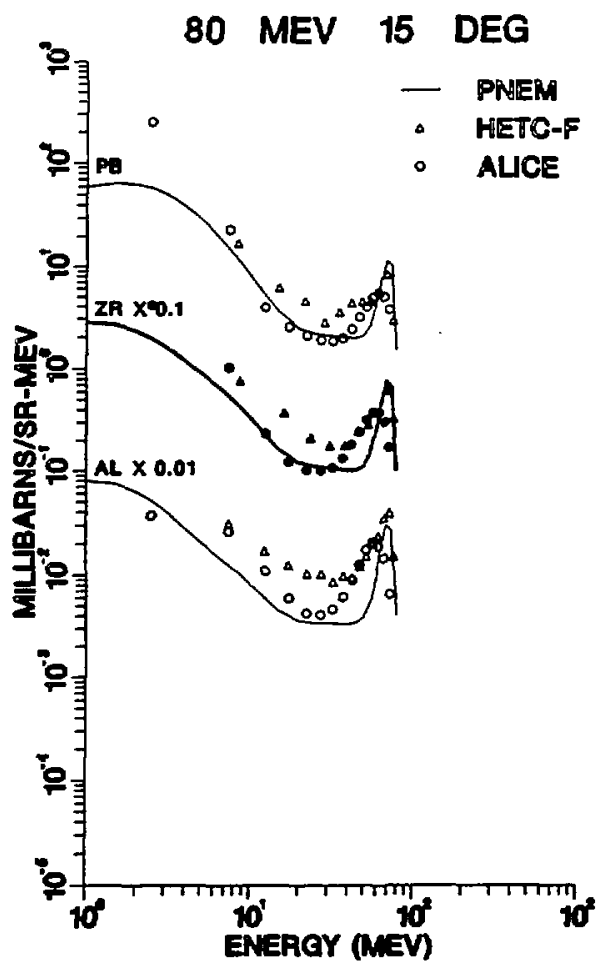

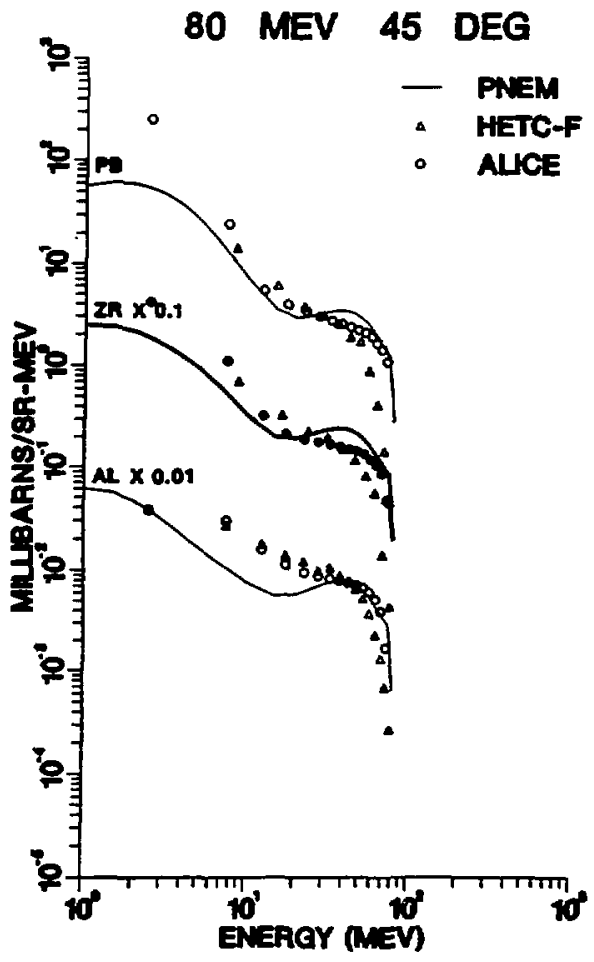


Fig. 7. cont.
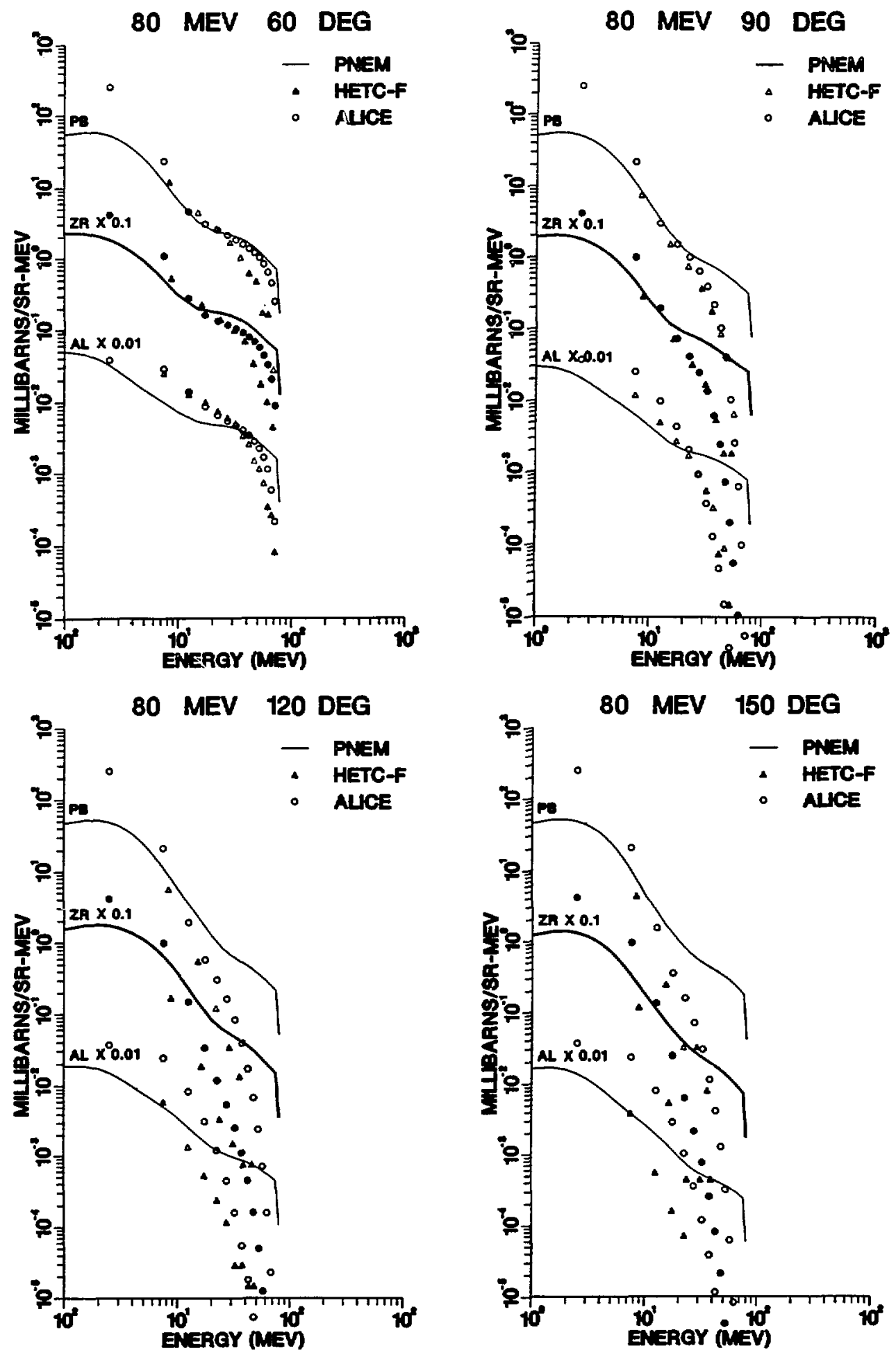
Fig. 8. Neutron emission spectra from $160 \mathrm{MeV}$ protons on Al-27, $\mathrm{Zr}-90$ and $\mathrm{Pb}-208$ at emission angles $15,30,45,60,90,120$ and 150 degrees.

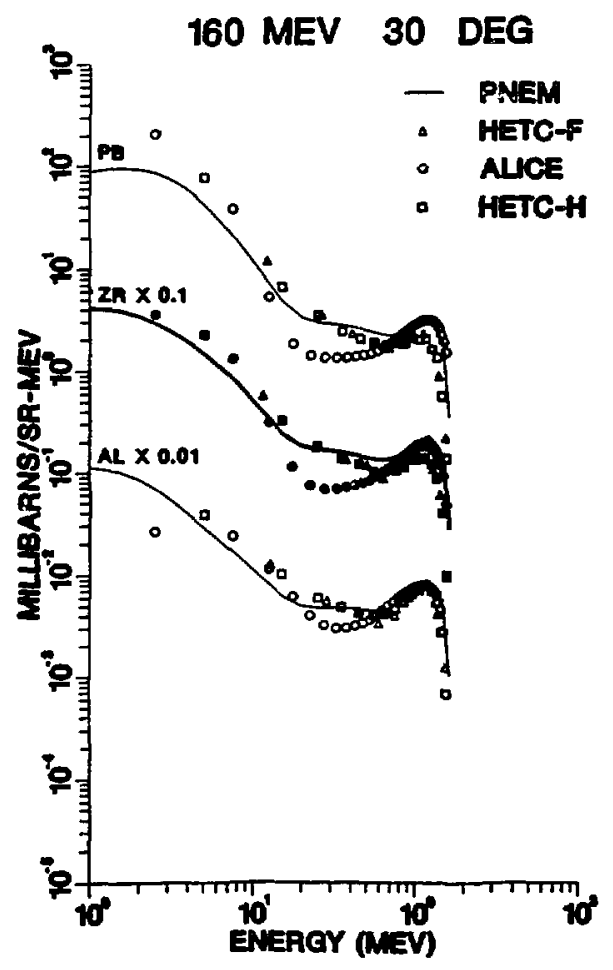

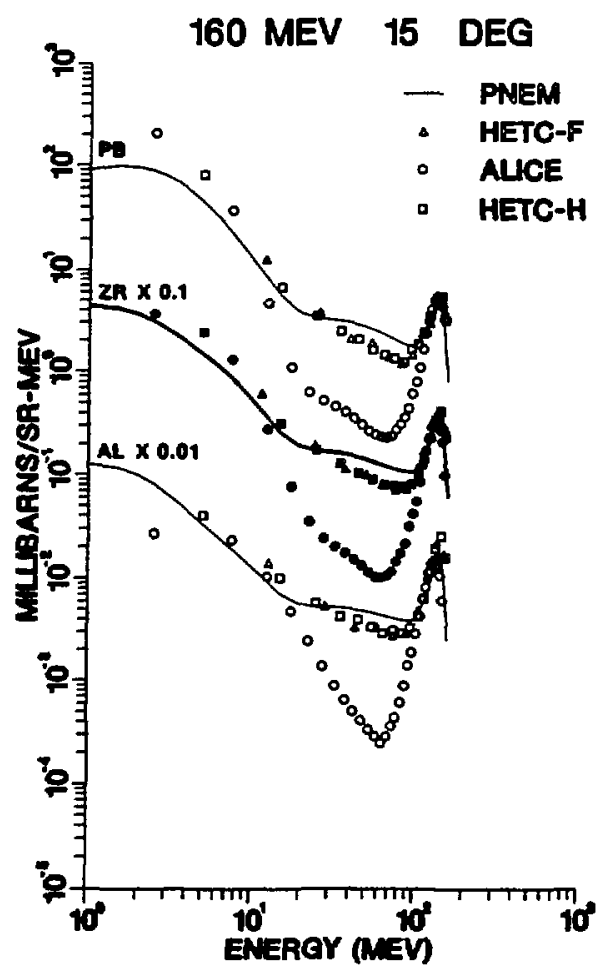

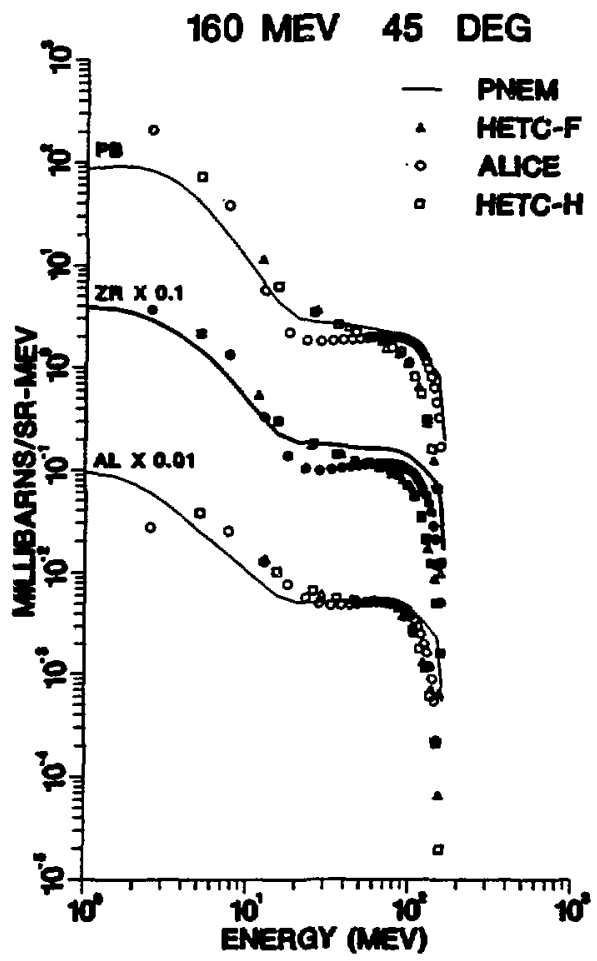


Fig. 8. cont.
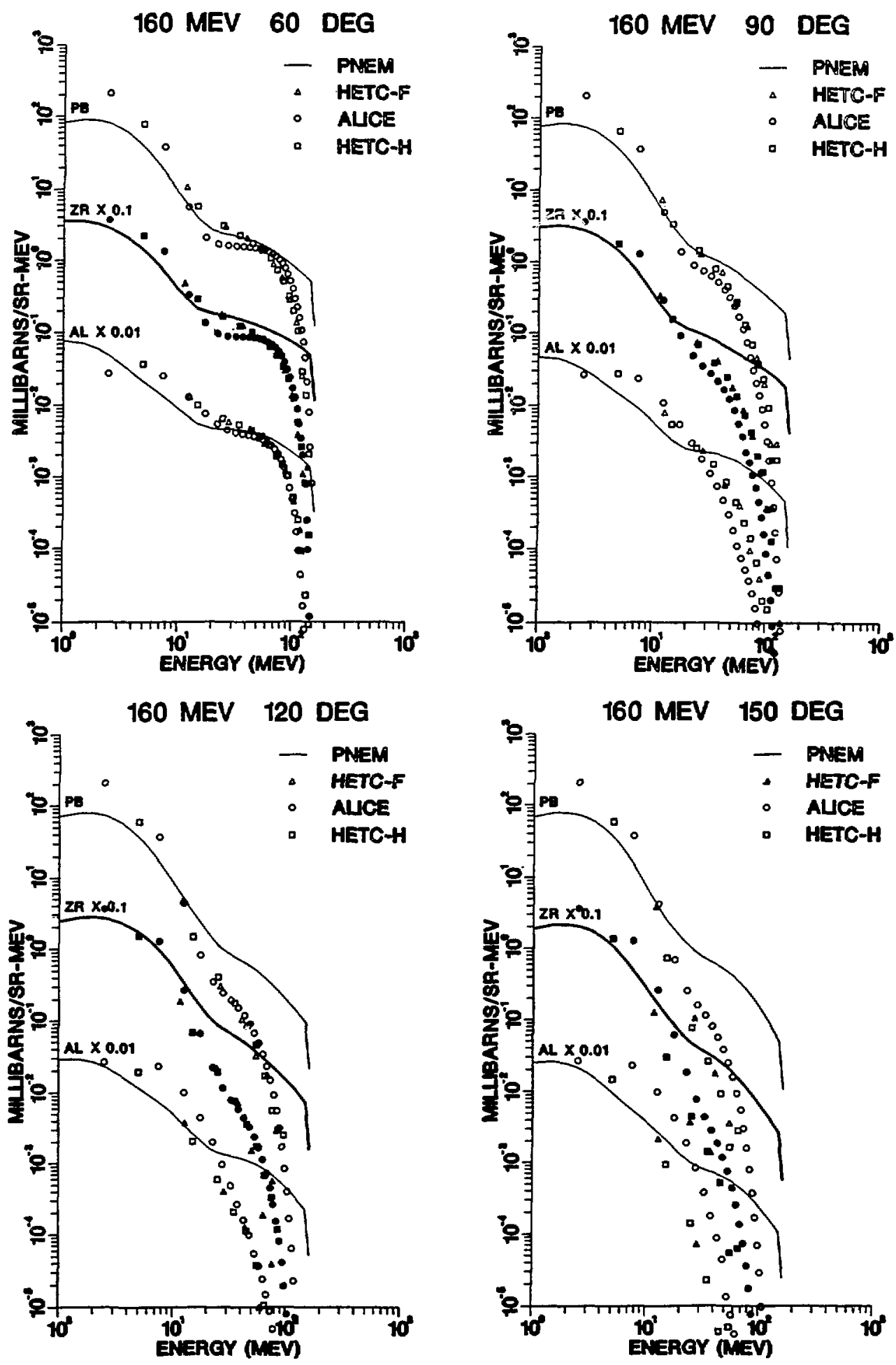
Fig. 9. Neutron energy spectra from $318 \mathrm{MeV}$ protons on A1-27, $\mathrm{Zr}-90$ and $\mathrm{Pb}-208$ at emission angles $15,30,45,60,90,120$ and 150 degrees.
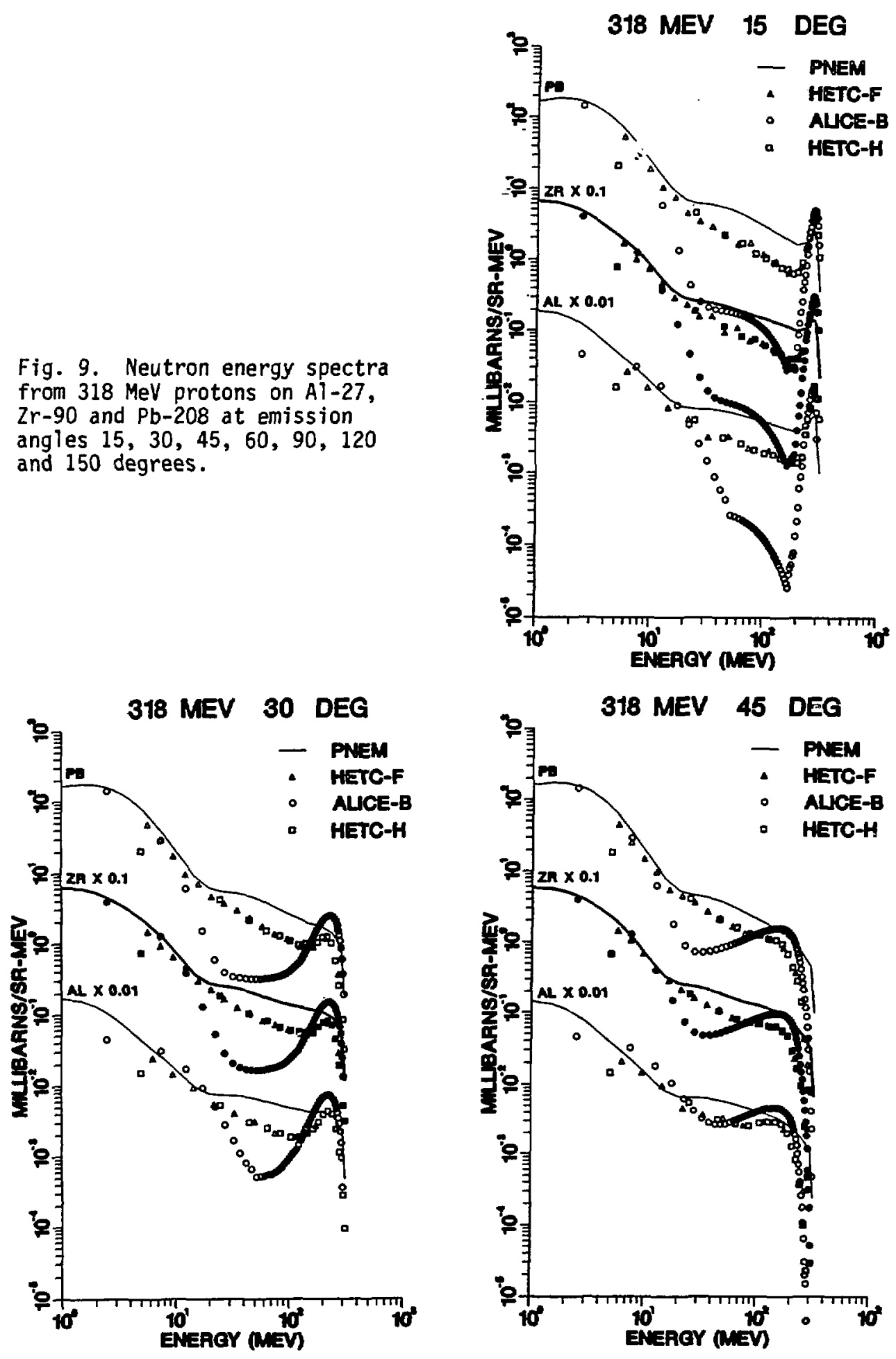
Fig. 9. cont.
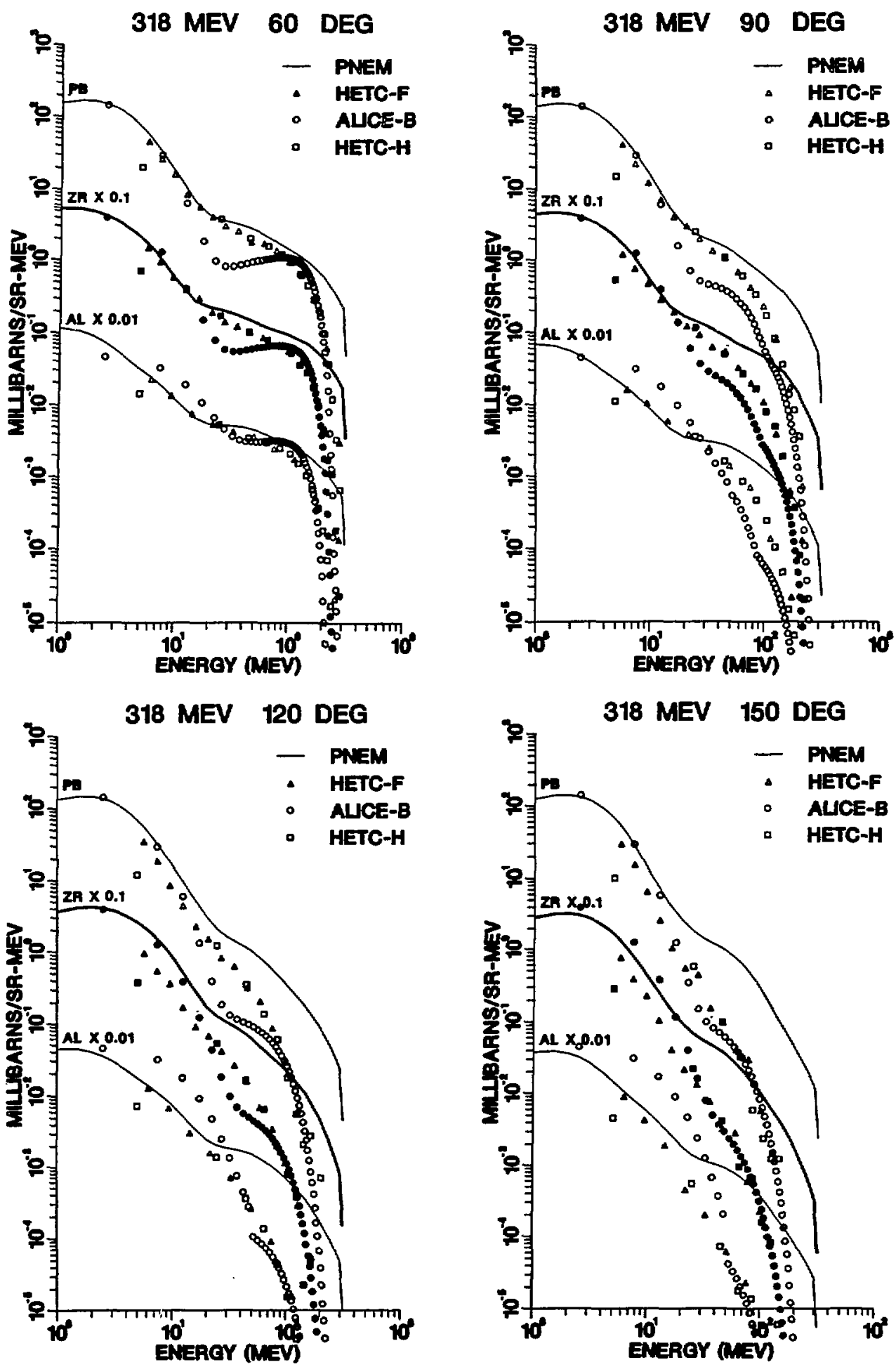
Fig. 10. Neutron energy spectra from $800 \mathrm{MeV}$ protons on A1-27, $\mathrm{Zr}-90$ and $\mathrm{Pb}-208$ at emission angles $15,30,45,60,90,120$ and 150 degrees.
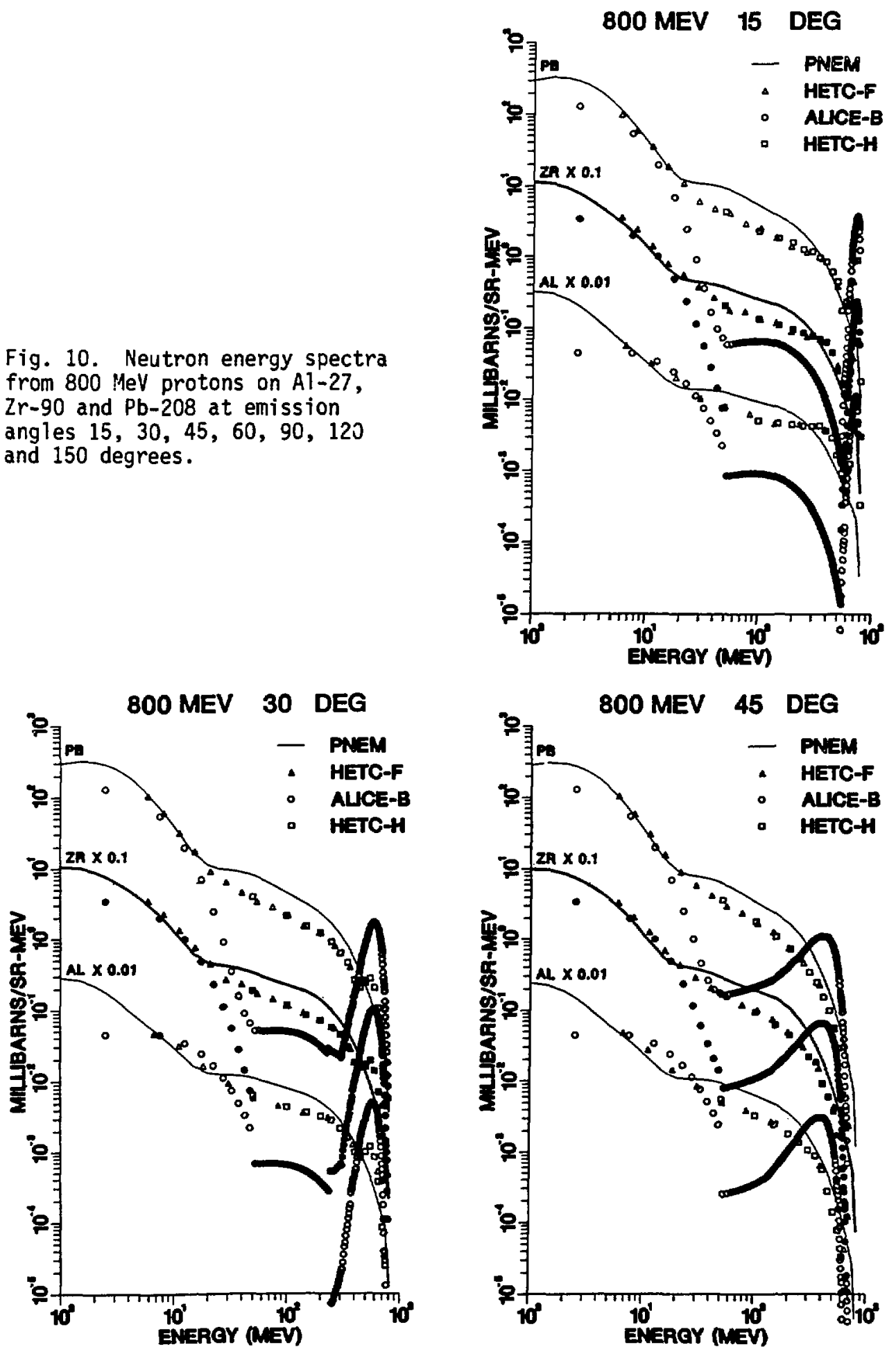
Fig. 10. cont.
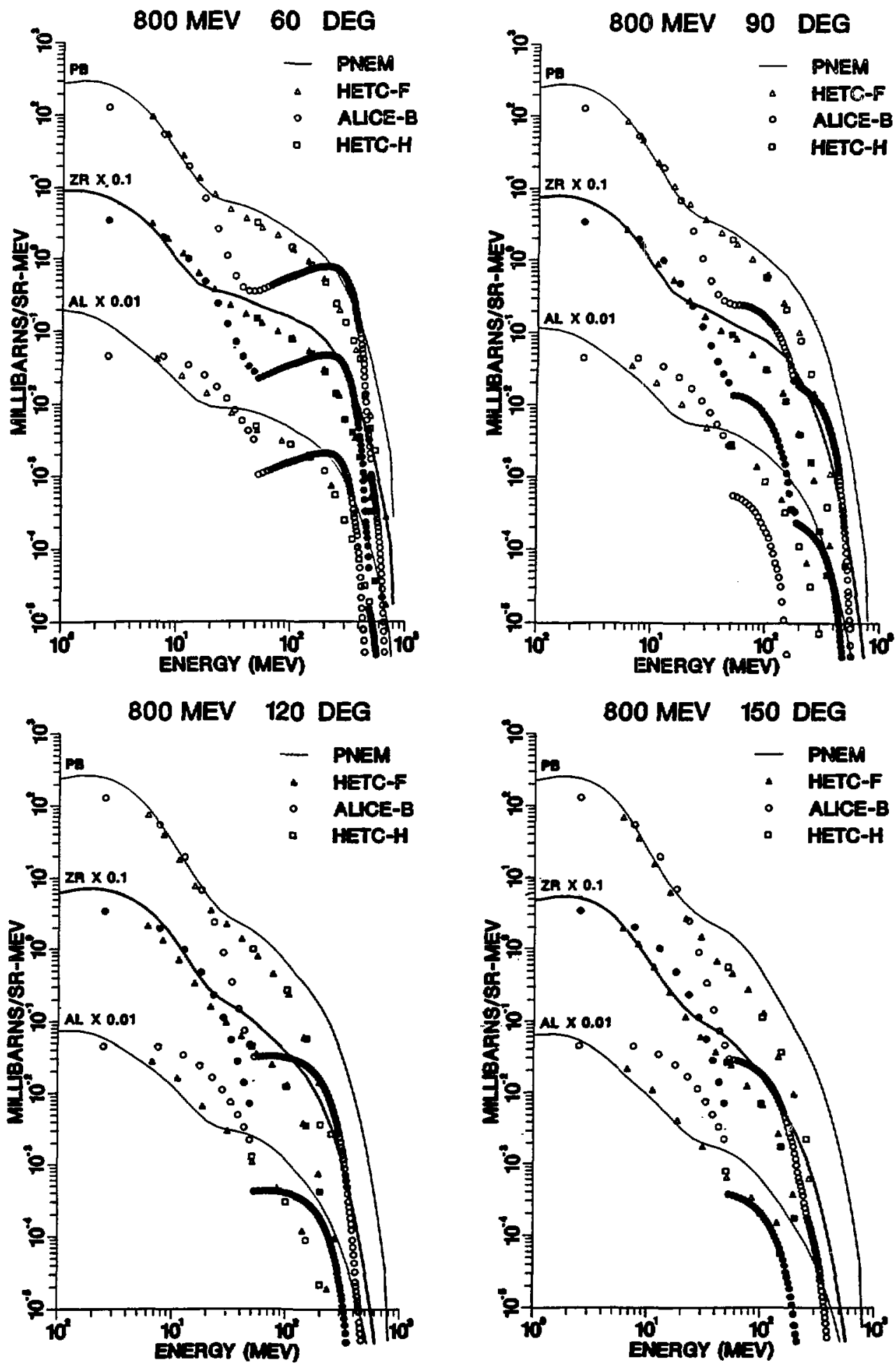
Fig. 11. Angular integrated neutron emission data at 35 , $80,160,318$ and $800 \mathrm{MeV}$ from A1-27, $\mathrm{Zr}-90$ and $\mathrm{Pb}-208$.

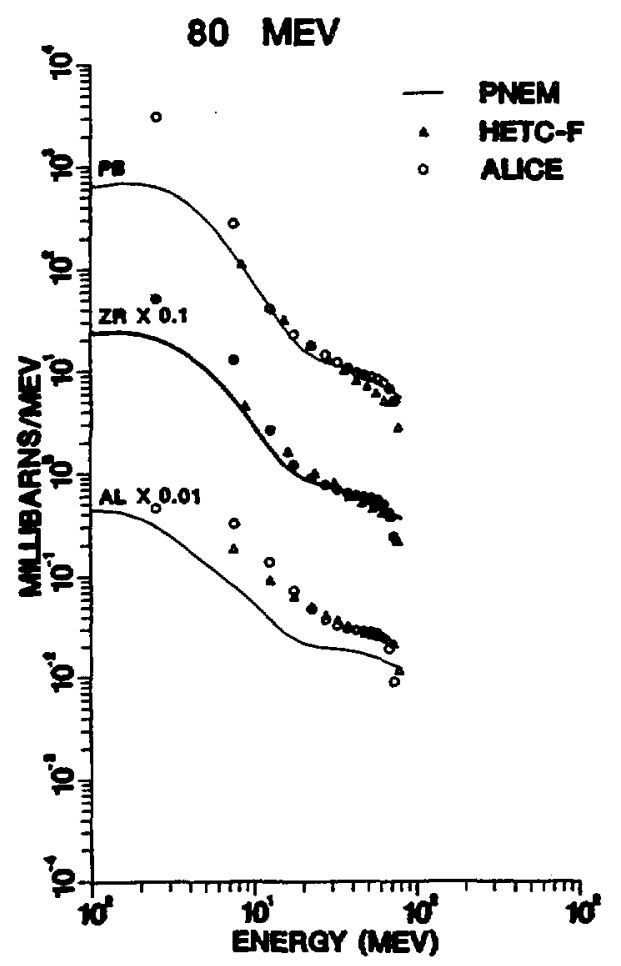

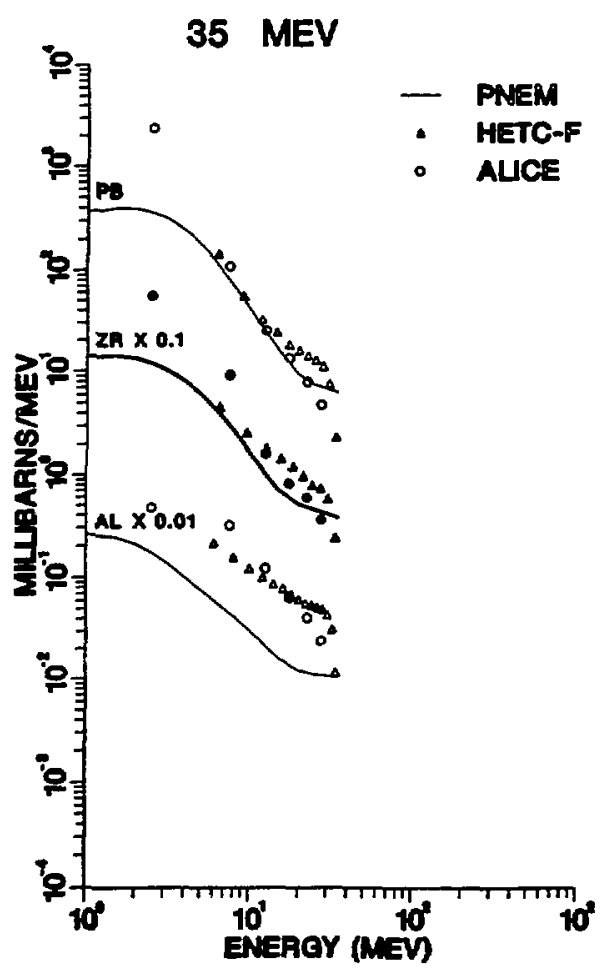

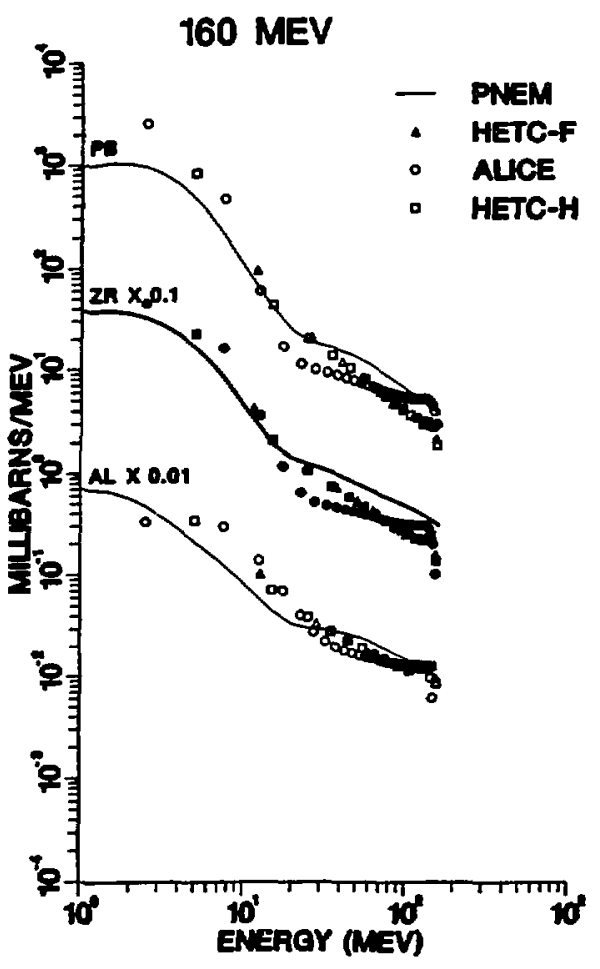


Fig. 11. cont.
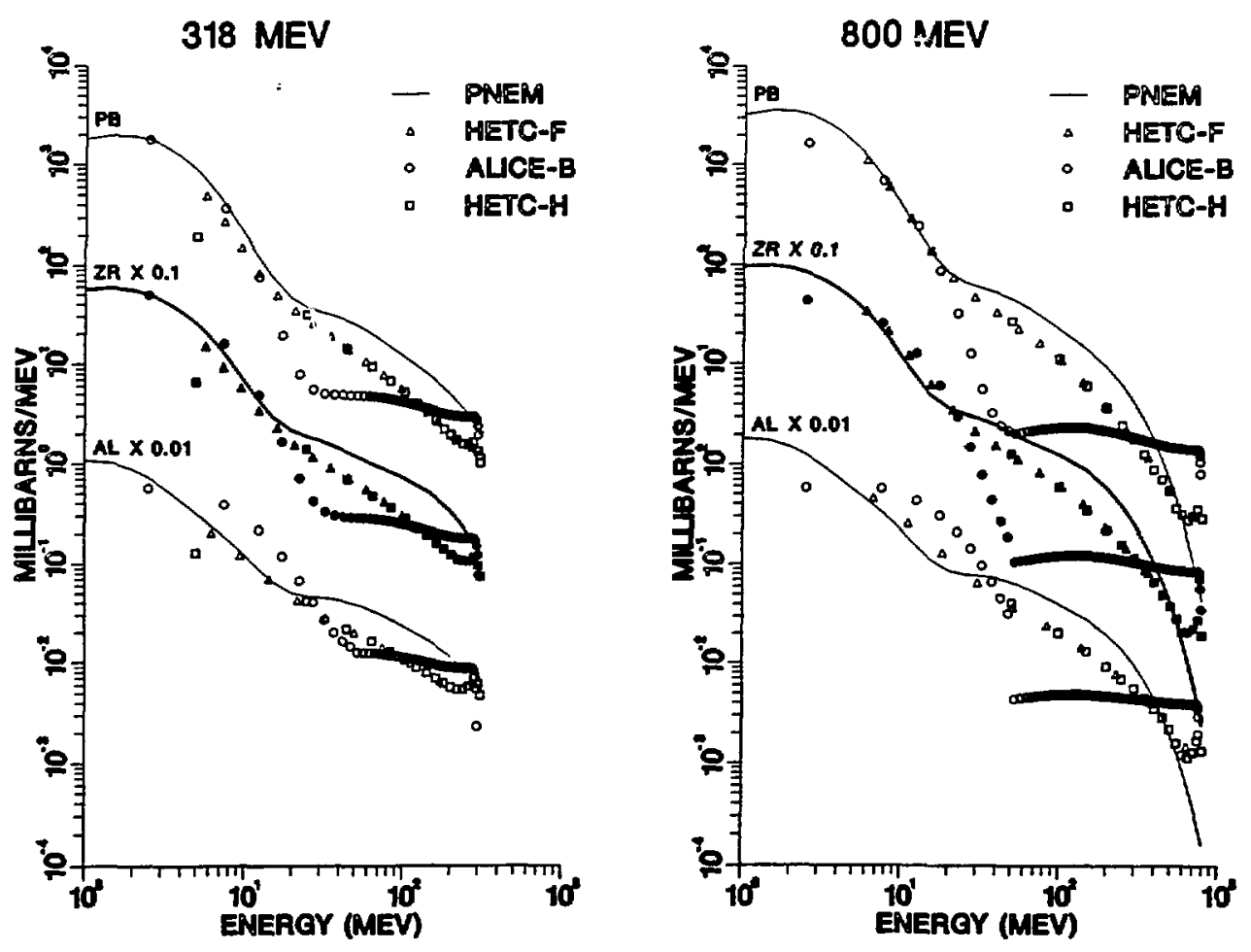